\title{
The incidence, clearance and persistence of non-cervical human papillomavirus infections: a systematic review of the literature
}

Sylvia Taylor ${ }^{1 *}$, Eveline Bunge ${ }^{2}$, Marina Bakker ${ }^{2}$ and Xavier Castellsaguée $e^{3,4}$

\begin{abstract}
Background: Human papillomavirus (HPV) vaccines were designed to prevent cervical cancer in women and their provision remains a major public health need. However, HPV is also a major cause of non-cervical anogenital and oropharyngeal cancers and the potential benefit of vaccination likely extends beyond cervical cancer.

Methods: A systematic literature search of PubMed (1995-2014) identified publications assessing the incidence, persistence, and clearance of non-cervical anogenital/oral HPV infections. Comparability with cervical HPV was assessed by identifying articles assessing the same or similar populations.

Results: Available data suggest high incidence rates of non-cervical HPV infection in men and women, with HPV-16 predominating in all sites. The incidence of high risk HPV per 100 person-years ranged from 11.4 to 72.9 for penile infections, 6.7-47.9 at other male genital sites, and 4.4-36.7 and 5.3-23.4 for anal infections in men and women, respectively. The incidence per 100 person-years of oral infection with any HPV type ranged from 5.7 to 6.7 in men and 6.8-39.6 in women. Within the limitations of the data, there was a general pattern of higher incidence and clearance of non-cervical genital HPV infections, compared to cervical infections. HIV status, circumcision, number of sex partners and partner HPV status significantly influenced high-risk HPV incidence/clearance at male anogenital sites. Few studies assessed risk factors for oral HPV.

Conclusions: Parallels appear to exist between the epidemiology of cervical and non-cervical HPV infections in terms of incidence, HPV-type distribution, and risk factors for infection. Available data suggest that non-cervical genital HPV infections may occur more frequently, with higher clearance rates, than cervical infections. More extensive studies could provide useful information for estimating vaccine impact, the wider cost-benefit of HPV vaccination, and guiding vaccination policy.
\end{abstract}

Trial registration: Not applicable, as systematic review of the literature.

Keywords: Human papillomavirus, Cervical, Penile, Oral, Vaginal, Systematic review, Incidence, Persistence, Clearance

\footnotetext{
* Correspondence: sylvia.m.taylor@gsk.com

'GSK Vaccines, 20, Avenue Fleming, Parc de la Noire Epine, B-1300 Wavre,

Belgium

Full list of author information is available at the end of the article
} 


\section{Background}

Cervical cancer is the fourth most common cancer affecting women worldwide, with an estimated 528,000 new cases and 266,000 related deaths in 2012 [1]. Cervical cancer develops following persistent infection with oncogenic (or high risk) types of the human papillomavirus (HPV), that includes types 16, 18, 31, 33, 35, 39, 45, 51, 52, 56, 58, 59, 66 [2]. Approximately $70 \%$ of cervical cancers are caused by HPV types 16 and 18, which along with several other high risk HPV types (HR-HPV), can be prevented by vaccination. However, the cancer-causing effects of HPV are not limited to the cervix; an estimated $50 \%$ of penile, $88 \%$ of anal, $43 \%$ of vulvar, $70 \%$ of vaginal, and 13-56 \% of oropharyngeal cancers are attributable to HPV, primarily HPV-16 typically followed by HPV-18 [3, 4]. Sharply increasing trends in HPV-related oropharyngeal cancers have been observed in some countries [5]. The incidence of cervical cancer is far higher than that of nongenital or oropharyngeal cancers, and the provision of HPV vaccines to prevent cervical cancer remains a public health priority. However, together non-cervical and oropharyngeal cancers represented approximately 80,000 new HPVrelated cancer cases worldwide in 2008, also signifying an important public health burden [6].

Available evidence from clinical trials indicates that current HPV vaccines can prevent vulval and vaginal and anal HPV infections, anogenital pre-cancers, and oral HPV infections [7] in women, and oral and anogenital infections and pre-cancer in men [7-14]. However, compared to vaccine efficacy/effectiveness data for cervical HPV infections and high-grade lesions, similar data for non-cervical HPV infections and lesions are scant. Moreover, our ability to measure the population level impact of HPV vaccination on non-cervical cancers is severely limited by a lack of systematic screening for non-cervical infections and pre-cancers, the relative rarity of these cancers, and for some, the absence of precursor lesions amenable to screening. The need to build ways of collecting such data nonetheless remains important to understand the full value of HPV vaccination, including cost-benefit, so that proper guidance can be given to vaccination policy. To this end, we conducted a systematic literature review to investigate the natural history of non-cervical HPV infections, and to identify parallels between the epidemiology of these infections and that of cervical infection. These data can inform on the design and scope of future studies of HPV vaccine effectiveness, aid in the interpretation of surveillance data, and point to knowledge gaps where further investigations may be warranted.

\section{Methods}

\section{Objectives}

The study objectives were to conduct a systematic review of the literature to describe the incidence, clearance and persistence of non-cervical HPV infections, including comparisons to cervical infections for which data were available for the same/or similar study population. We also examined risk factors for incident and persistent non-cervical HPV infections.

\section{Search strategy and selection criteria}

A systematic review of the literature in PubMed used three search strings (Supplementary material) to identify noncervical anogenital and oral HPV infections and was limited to studies published in English between 01 January 1995 and 12 July 2014. We used specific searches to obtain articles on cervical HPV infections reporting comparable estimates of incidence, clearance, or persistence in the same or similar populations and using comparable HPV testing methods as in the articles on non-cervical HPV infections. Articles were included if they contained information on incidence, persistence, clearance or duration of non-cervical HPV infections, risk factors for non-cervical $\mathrm{HPV}$ infections or risk groups for incidence of noncervical infections. Articles were excluded if they reported diagnostic test research, if they were case studies, letters to the editor, editorials or comments, literature reviews, or meta-analyses presenting no original data. Articles were also excluded if no information was listed in the inclusion criteria, or if they reported data based on testing of selfcollected vaginal samples only. PRISMA guidelines were followed for the report. Independent review in duplicate was undertaken for $30 \%$ of screened titles and abstracts and for $10 \%$ of full text articles. Article selection, data extraction, assessment of risk factors and quality control procedures are provided in Additional file 1.

During the search for cervical cohorts, studies were found which used self-collected vaginal samples but in which the samples were referred to as 'cervical' swabs. These studies were not identified with the original noncervical search string that did not include the term 'cervical'. We did not include studies when vaginal samples had been self-collected, because the original search may have missed studies that assessed self-collected vaginal samples, and because we could not be certain that they represented vaginal-only infections. For similar reasons cervical samples collected using cervical vaginal lavage in D'Souza et al. [15] were also excluded because we could not be certain that they represented cervical-only infections.

For ease of comparability, incidence, clearance and persistence rates were recalculated, if not provided, per 100 person-years, and all estimates of median (or mean if median not reported) time-to-clearance (or duration of infection) were calculated in terms of months. For example, conversion from 1000 person-months to 100 person-years values was done by multiplying by 12 and dividing by 10. All estimates of median time-to-clearance were calculated in terms of months (dividing days by 30.5 ). 
When confidence intervals $(\mathrm{CI})$ were not already provided and there was sufficient data, we used the exact method to calculate $95 \% \mathrm{CI}$ for proportions.

Ethics approval was not required for this study.

\section{Definitions}

The definition of HR-HPV was study specific. The HRHPV types applicable to individual studies are provided in Additional file 2: Table S1.

Clearance, incidence, persistence, and duration of infection were defined broadly to capture the most studies: For individual HPV types (e.g., HPV-16), incidence was defined as at least one positive test for that HPV type following at least one negative test for that type. Clearance of infection was defined as a negative test for the individual HPV type following a positive test for that type.

Type-specific incidence of any HPV or HR-HPV was defined as a positive test for at least one HPV type or HRHPV type not detected at baseline or other previous visit. For non-type-specific incidence, subjects were required to have tested negative for any HPV DNA or any HR-HPV DNA at baseline. We considered this to be non-typespecific incidence even if the HPV type(s) associated with the incident infection was known. In addition, for studies conducting multiple follow-up visits, incidence rates could be calculated by: 1) censoring subjects at the time of the first positive test (first acquired incidence); or 2) considering subjects to be at risk throughout the follow-up period and counting each visit where at least one new type was detected as an event (total acquired incidence).

Type-specific clearance of any HPV or HR-HPV was defined as a negative test for all HPV types or HR-HPV types detected at baseline (type-specific clearance of prevalent infection) or for all newly detected during follow-up (type-specific clearance of incident infection). Non-type-specific clearance was defined as at least one negative test for any HPV DNA or any HR-HPV DNA following a positive test for any HPV DNA or HR-HPV DNA at baseline or during follow-up.

Persistence was defined as at least two sequentially positive tests for at least one specific HPV type or HRHPV type at least three months apart. Definitions for type-specific and non-type specific duration of infection followed those for clearance of infection.

For estimates related to any HPV and HR-HPV, calculations were required to be based on the total number of subjects rather than total number of infections as the denominator.

\section{Results}

\section{Search results}

We identified 38 articles from 25 unique study cohorts with relevant data on non-cervical anogenital or oral HPV infections (Table 1). A further 6 articles were identified that assessed cervical HPV infection in the same/similar populations. HPV-testing methods are summarised in Additional file 2: Table S1. Three articles were excluded due to unclear methods. Articles excluded due to improper testing methods or sample type were included under 'no relevant data'.

Median follow-up times varied between 6.7 and 84.5 months, with all but 8 studies conducting at least two follow-up visits at intervals ranging between 2 and 12 months. Among the 31 studies reporting data on incidence of any HPV or any HR-HPV, 10 (32\%) used type-specific definitions which counted any newly detected type as an incident infection and 17 (55\%) used non-type-specific definitions that required subjects to be negative for any HPV or any HR-HPV at baseline; an additional 4 studies reported data on incidence of individual HPV types only. A total of 31 studies were known to have multiple follow-up visits. Among the 31, 20 assessed incidence of any HPV or HR-HPV: 13/20 (65 \%) estimated incidence based on time to the first acquired infection; $3 /$ 20 (15\%) considered each new infection as an event; for $2 / 20$ it was unclear (10 \%); $1 / 20$ (5\%) used both methods; and $1 / 20(5 \%)$ did not conduct any survival analysis. Among the 29 studies reporting data on HPV clearance, type-specific definitions were used more consistently, with only 5 (17\%) using non-type-specific definitions. Three studies $(10 \%)$ required at least two consecutive negative test results to define an infection as having cleared.

\section{Penile HPV infections Incidence}

In studies conducted in Africa, HR-HPV incidence was higher in human immunodeficiency virus positive (HIV + ve) men (42.0-72.9/100 person-years) than HIV negative (HIV-ve) men (19.7-32.9/100 person-years) (Table 2). Studies in Spain reported higher HPV incidence in HIV + ve men who have sex with men (MSM) than in men who have sex with women (MSW) (11.6 versus 5.1/100 personyears), but similar incidences of HPV-16 and HPV-18 infection. Genotype-specific incidence was highest for HPV-16 in at least one study subgroup in 7/9 studies (HIV + ve, HIV-ve, circumcised, uncircumcised and MSW) [8, 16-21].

In three articles that reported age-specific incidence of HPV infection the highest incidence was reported in the youngest age group studied ( $<30$ or $15-24$ year olds) $[18,19,22]$.

\section{Clearance and persistence}

The rate of clearance of incident HR-HPV infection was 123.9/100 person-years (Table 2), with HPV-33 clearing most rapidly and HPV-58 least rapidly [22].

The clearance rate of prevalent penile infections ranged between 23.6 and 114.1/100 person-years for any HPV [19, 20, 23]. Clearance of HR-HPV was 114.7/100 person-years in HIV + ve men and 170.2/100 person- 
Table 1 Summary of 38 articles included in the review

\begin{tabular}{|c|c|c|c|c|c|c|c|c|}
\hline $\begin{array}{l}\text { Author, study } \\
\text { location (ref) }\end{array}$ & Study year & Population & Age & Number & $\begin{array}{l}\text { Follow-up period } \\
\text { (timing of follow-up visits) }\end{array}$ & $\begin{array}{l}\text { Mean or median } \\
\text { follow-up }\end{array}$ & Anatomical site & $\begin{array}{l}\text { Outcomes } \\
\text { (definition } \\
\text { used for } \\
\text { any/HR-HPV) }\end{array}$ \\
\hline Tobian, Uganda [19] & $2003-2007$ & HIV + ve/-ve men & $15-49$ & 999 & 2 years $(6,12,24 \mathrm{~m})$ & Mean $14.4 \mathrm{~m}$ & Penile & $\begin{array}{l}\text { Inc (TS, total) } \\
\text { Clr (TS) }\end{array}$ \\
\hline Kjaer, Denmark [63] & $2000-2003$ & Military servicemen & $18-19$ & 374 & $6 \mathrm{~m}(6 \mathrm{~m})$ & Range $5.4-7.8 \mathrm{~m}$ & Penile & Inc (NTS) \\
\hline Backes, Kenya [17] & $2002-2005$ & Uncircumcised men & $17-24$ & 966 & 1 year $(6,12 \mathrm{~m})$ & Median $12.1 \mathrm{~m}$ & Penile & Inc (NTS, first) \\
\hline $\begin{array}{l}\text { Mbulawa, South } \\
\text { Africa [22] }\end{array}$ & Not reported & HIV + ve/-ve men & $19-67$ & 486 & 2 years $(6,12,18,24 \mathrm{~m})$ & - & Penile & $\begin{array}{l}\text { Inc (TS, first) } \\
\operatorname{Clr}(T S)^{\mathrm{a}}\end{array}$ \\
\hline Videla, Spain [23] & 2005-2009 & $H I V$ + ve men & $20->69$ & 733 & 5 years (annual) & Median $24 \mathrm{~m}$ & Penile, anal, oral & $\begin{array}{l}\text { Inc (NTS, first) } \\
\text { Clr (NTS) }\end{array}$ \\
\hline $\begin{array}{l}\text { Wikström A, } \\
\text { Sweden [21] }\end{array}$ & Not reported & $\begin{array}{l}\text { MSW, sexually active } \\
\text { without clinical HPV }\end{array}$ & $18-54$ & 235 & (1-5 visits) & $\begin{array}{l}\text { Mean } 3.5 \mathrm{~m} \\
\text { between visits }\end{array}$ & Penile & $\begin{array}{l}\text { Inc (TS) } \\
\text { CIr/Pers (TS) }\end{array}$ \\
\hline Gray, Uganda [18] & $2003-2006$ & HIV-ve married men & $15-49$ & 840 & $24 \mathrm{~m}(6,12,24 \mathrm{~m})$ & - & Penile & $\begin{array}{l}\text { Inc } \\
\text { Clr }\end{array}$ \\
\hline Serwadda, Uganda [16] & $2003-2007$ & $\mathrm{HIV}$ + ve men & $15-49$ & 210 & $24 \mathrm{~m}(24 \mathrm{~m})$ & - & Penile & $\begin{array}{l}\operatorname{lnc}(\mathrm{TS}) \\
\mathrm{Clr}\end{array}$ \\
\hline Grabowski, Uganda [24] & 2003-2007 & HIV + ve/-ve men & $15-49$ & 936 & $24 \mathrm{~m}(6,12,24 \mathrm{~m})$ & - & Penile & Pers (TS) \\
\hline $\begin{array}{l}\text { Giuliano, Brazil, } \\
\text { Mexico, USA [53] }\end{array}$ & 2005-2009 & HIV-ve men & $18-70$ & 1159 & 4 years (every $6 \mathrm{~m}$ ) & Median 27.5 m & Penile & $\begin{array}{l}\text { Inc (NTS, first) } \\
\operatorname{Clr}(\mathrm{TS})\end{array}$ \\
\hline Darwich, Spain [20] & $2005-2009$ & HIV + ve men & $20->69$ & 606 & 5 years (annual) & Median $24 \mathrm{~m}$ & Penile, anal & $\begin{array}{l}\ln c^{b} \\
\text { Clr }\end{array}$ \\
\hline $\begin{array}{l}\text { de Pokomandy, } \\
\text { Canada [38] }\end{array}$ & $2002-2007$ & HIV + ve MSM & $21-66$ & 247 & 3 years (every 6 m) & Mean $30.8 \mathrm{~m}$ & Anal & $\begin{array}{l}\text { Inc } \\
\text { Clr }\end{array}$ \\
\hline $\begin{array}{l}\text { Nyitray, Brazil, } \\
\text { Mexico, USA [35] }\end{array}$ & $2005-2009$ & Men & $18-70$ & $\begin{array}{l}\text { MSM:156 } \\
\text { MSW:954 }\end{array}$ & $6 \mathrm{~m}(6 \mathrm{~m})$ & Median 6.7 m & Anal & $\begin{array}{l}\text { Inc (TS) } \\
\text { Clr (TS) } \\
\text { Pers (TS) }\end{array}$ \\
\hline $\begin{array}{l}\text { Goodman, } \\
\text { Hawaii, USA [36] }\end{array}$ & 1998-2003 & $\begin{array}{l}\text { Sexually active } \\
\text { women }\end{array}$ & $18-85$ & 431 & 5 years (every $4 \mathrm{~m}$ ) & Mean $16 \mathrm{~m}$ & Anal & $\begin{array}{l}\text { Inc (NTS, first) } \\
\text { Clr (TS) }\end{array}$ \\
\hline $\begin{array}{l}\text { Shvetsov, } \\
\text { Hawaii [39] }\end{array}$ & 1998-2003 & $\begin{array}{l}\text { Sexually active } \\
\text { women }\end{array}$ & $18-85$ & 431 & 5 years (every 4 m) & Mean $16 \mathrm{~m}$ & Anal & $\mathrm{Clr}(\mathrm{TS})$ \\
\hline Moscicki, USA [40] & $1990->2004$ & $\begin{array}{l}\text { Sexually active } \\
\text { women }\end{array}$ & $13-21$ & 75 & 3-9.5 years (every $4 \mathrm{~m}$ ) & Mean $84.5 \mathrm{~m}$ & Anal & Clr (TS, 2+ neg) \\
\hline Mullins, USA [37] & $1996-2001$ & $\begin{array}{l}\text { HIV + ve and } \\
\text { HIV-ve at risk }\end{array}$ & $12-18$ & 496 & 6 years (annual) & Mean $22.4 \mathrm{~m}$ & Anal & Inc (NTS, first) \\
\hline Glick, USA [33] & 2009-2010 & MSM & $16-30$ & 94 & 1 year (every $6 \mathrm{~m}$ ) & - & Anal & $\begin{array}{l}\text { Inc (NTS, unclear) } \\
\text { CIr (TS) }\end{array}$ \\
\hline Hernandez, USA [34] & $1998-2000$ & HIV + ve MSM & Mean 45 & 369 & 2 years (every $6 \mathrm{~m}$ ) & - & Anal & Inc (TS, total) \\
\hline
\end{tabular}


Table 1 Summary of 38 articles included in the review (Continued)

\begin{tabular}{|c|c|c|c|c|c|c|c|c|}
\hline Lu, Arizona USA [31] & $2003-2005$ & Men & $18-44$ & 285 & 2 years (every $6 \mathrm{~m}$ ) & Median $15.5 \mathrm{~m}$ & $\begin{array}{l}\text { Male genital } \\
\text { (shaft, coronal } \\
\text { sulcus, glans, } \\
\text { scrotum) }\end{array}$ & $\begin{array}{l}\text { Inc (TS, total) } \\
\text { Clr (TS) }\end{array}$ \\
\hline $\begin{array}{l}\text { Hernandez, } \\
\text { Hawaii [59] }\end{array}$ & 2004-2006 & Circ, UnC men & $18-79$ & 357 & 2.5 years (every 2 m) & Mean 14 m & $\begin{array}{l}\text { Male genital } \\
\text { (coronal sulcus, } \\
\text { glans, shaft, } \\
\text { scrotum) }\end{array}$ & Clr (TS, 2+ neg) \\
\hline $\begin{array}{l}\text { Giuliano, } \\
\text { Arizona USA [25] }\end{array}$ & 2003-2005 & Men & $18-44$ & 290 & 2 years (every $6 \mathrm{~m}$ ) & Mean $15.5 \mathrm{~m}$ & $\begin{array}{l}\text { Male genital } \\
\text { (coronal sulcus, } \\
\text { glans, shaft, } \\
\text { scrotum) }\end{array}$ & $\begin{array}{l}\text { Inc (NTS, first \& total) } \\
\text { Pers (NTS) } \\
\text { Clr (NTS) }\end{array}$ \\
\hline $\begin{array}{l}\text { Morales, } \\
\text { Mexico [26] }\end{array}$ & 2003-2004 & MSW & Median 36 & 351 & 3 years (every $4 \mathrm{~m}$ ) & Median 19.8 m & $\begin{array}{l}\text { Male genital } \\
\text { (scrotum, shaft, } \\
\text { balano-preputial } \\
\text { groove, urinary } \\
\text { meatus) }\end{array}$ & $\begin{array}{l}\text { Inc (NTS, unclear) } \\
\text { Clr (TS) }\end{array}$ \\
\hline Partridge, USA [29] & 2003-2006 & $\begin{array}{l}\text { MSW university } \\
\text { students }\end{array}$ & $18-20$ & 240 & 3 years (every $4 \mathrm{~m}$ ) & Median 12.9 m & $\begin{array}{l}\text { Male genital } \\
\text { (glans \&urethral } \\
\text { meatus, shaft, } \\
\text { scrotum) }\end{array}$ & Inc (NTS, first) \\
\hline Lajous, Mexio [27] & $2000-2003$ & Male soldiers & $16-40$ & 336 & 1 year (1 year) & - & $\begin{array}{l}\text { Male genital } \\
\text { (shaft, coronal } \\
\text { sulcus, scrotum } \\
\text { urethral meatus) }\end{array}$ & $\begin{array}{l}\text { Inc (NTS) } \\
\text { Clr (TS) } \\
\text { Pers (TS) }\end{array}$ \\
\hline $\begin{array}{l}\text { Albero, Brazil, } \\
\text { Mexico, USA [28] }\end{array}$ & $2005-2009$ & HIV-ve men & $18-70$ & 4033 & 4 years (every $6 \mathrm{~m}$ ) & Median 17.5 m & $\begin{array}{l}\text { Male genital } \\
\text { (shaft, coronal } \\
\text { sulcus, glans, } \\
\text { scrotum) }\end{array}$ & $\begin{array}{l}\text { Inc (NTS, first) } \\
\text { Clr (TS, 2+ neg) }\end{array}$ \\
\hline Winer, USA [43] & 1990-2000 & $\begin{array}{l}\text { Female university } \\
\text { students }\end{array}$ & $18-20$ & 444 & 10 year (every 4 m) & Mean $41.2 \mathrm{~m}$ & Vulvovaginal & Inc (NTS, first) \\
\hline Edelstein, USA [45] & 2008-2010 & Male university students & Median 20 & 212 & 1.5 years (every 4 m) & Median 10.7 m & Oral (rinse/OP swab) & Inc (NTS, first) \\
\hline Kero, Finland [48] & Not reported & $\begin{array}{l}\text { Male partners of } \\
\text { pregnant women }\end{array}$ & $19-46$ & 131 & $\begin{array}{l}7 \text { years }(2,6,12,24 \\
36 \mathrm{~m} \& 7 \text { years })\end{array}$ & $\begin{array}{l}\text { Mean at visit 1: } \\
1.8 \mathrm{~m} \text {, visit 2: } 5.9 \mathrm{~m} \text {, } \\
\text { visit } 3: 12.1 \mathrm{~m}, \\
\text { visit 4: } 24.7 \mathrm{~m}, \\
\text { visit 5: } 36.8 \mathrm{~m}, \\
\text { visit 6: } 77.0 \mathrm{~m}\end{array}$ & Oral (scraping) & Inc \\
\hline Kero, Finland [30] & $2006-2008$ & $\begin{array}{l}\text { Pregnant women } \\
\text { and their male } \\
\text { partners }\end{array}$ & $\begin{array}{l}\text { Women: 19-46 } \\
\text { Men: 20-52 }\end{array}$ & $\begin{array}{l}46 \text { women, } \\
46 \text { men }\end{array}$ & 7 years (7 years) & - & $\begin{array}{l}\text { Oral (scraping), } \\
\text { male genital } \\
\text { (penis/urethra) }\end{array}$ & $\begin{array}{l}\text { Inc (NTS) } \\
\text { Clr (TS) }\end{array}$ \\
\hline Kero, Finland [46] & Not reported & $\begin{array}{l}\text { Males partners of } \\
\text { pregnant women }\end{array}$ & $20-52$ & 129 men & $\begin{array}{l}7 \text { years }(2,6,12,24,36, \\
7 \text { years })\end{array}$ & $\begin{array}{l}\text { Mean in } 74 \\
\text { HPV + ve } 43.8 \mathrm{~m}\end{array}$ & Oral (scraping) & $\begin{array}{l}\text { Inc (NTS, first) } \\
\operatorname{Clr}(\mathrm{TS})\end{array}$ \\
\hline
\end{tabular}


Table 1 Summary of 38 articles included in the review (Continued)

\begin{tabular}{|c|c|c|c|c|c|c|c|c|}
\hline $\begin{array}{l}\text { Louvanto, } \\
\text { Finland [51] }\end{array}$ & Not reported & $\begin{array}{l}\text { Women with } \\
\text { persistent cervical } \\
\text { HPV ( } \geq 24 \text { months) } \\
\text { and HPV-ve controls }\end{array}$ & Mean 25.2-26.4 & $\begin{array}{l}43 \text { cases } \\
52 \text { controls }\end{array}$ & 6 years (possibly annual) & $\begin{array}{l}\text { Mean } 65.2 \text { m (cases) } \\
38.4 \text { m (controls) }\end{array}$ & Oral (scraping) & $\mathrm{Clr}(\mathrm{TS})$ \\
\hline Rintala, Finland [50] & $2006-2008$ & $\begin{array}{l}\text { Pregnant women } \\
\text { and their male } \\
\text { partners }\end{array}$ & $\begin{array}{l}\text { Women: 19-46 } \\
\text { Men: 20-52 }\end{array}$ & $\begin{array}{l}331 \text { women, } \\
131 \text { men }\end{array}$ & 2 years $(2,6,12,24 \mathrm{~m})$ & $\begin{array}{l}\text { Mean } 26.9 \text { m (women) } \\
25.9 \text { m (men) }\end{array}$ & Oral (scraping) & $\begin{array}{l}\text { Inc (NTS, first) } \\
\text { Clr (NTS) }\end{array}$ \\
\hline Pickard, USA [49] & 2009-2010 & University students & 18-30 & 1000 & $3 \mathrm{~m}(3 \mathrm{~m})$ & - & Oral (rinse) & $\begin{array}{l}\text { Inc (TS) } \\
\text { Clr (TS) } \\
\text { Pers (TS) }\end{array}$ \\
\hline D'Souza, USA [15] & 2004-2005 & $\begin{array}{l}\text { HIV + ve and high-risk } \\
\text { HIV-ve adult women }\end{array}$ & Not reported & 199 & $6 \mathrm{~m}(6 \mathrm{~m})$ & - & Oral (rinse) & $\begin{array}{l}\text { Inc (TS) } \\
\text { Clr (NTS) }\end{array}$ \\
\hline Kurose, Japan [52] & 2000-2002 & Healthy volunteers & $3-85$ & 662 & 2.5 years ( 2.5 years) & - & Oral (scraping) & $\begin{array}{l}\text { Clr (TS) } \\
\text { Pers (TS) }\end{array}$ \\
\hline Darwich, Spain [47] & $2005-2009$ & HIV + ve men & Median 41 & 733 & 5 years (annual) & Median $24 \mathrm{~m}$ & Oral (scraping/rinse) & Clr (TS) \\
\hline $\begin{array}{l}\text { Kreimer, Brazil, } \\
\text { Mexico, USA [44] }\end{array}$ & 2007-2009 & HIV-ve men & $18-70$ & 1626 & 4 years (every 6 m) & Median 12.7 m & Oral (rinse) & $\begin{array}{l}\text { Inc (NTS, first) } \\
\text { Clr (TS) }\end{array}$ \\
\hline
\end{tabular}


Table 2 Incidence and clearance of penile and other male genital HPV infections

\begin{tabular}{|c|c|c|c|c|c|c|c|c|c|c|c|c|}
\hline \multirow[t]{2}{*}{ Author (ref) } & \multirow{2}{*}{$\begin{array}{l}\text { Population, } \\
\text { age (years) }\end{array}$} & \multicolumn{5}{|l|}{ Incidence } & \multicolumn{6}{|l|}{ Clearance } \\
\hline & & Unit & Any HPV & $\mathrm{HR}$ & 16 & 18 & $\begin{array}{l}\text { Type of } \\
\text { Infection }\end{array}$ & $\begin{array}{l}\text { Unit of } \\
\text { clearance }\end{array}$ & Any HPV & $\mathrm{HR}$ & 16 & 18 \\
\hline \multicolumn{13}{|l|}{ Penile } \\
\hline Tobian [19] & $\begin{array}{l}\text { HIV-ve, 15-49 } \\
\text { HIV + ve,15-49 }\end{array}$ & per 100py & & $\begin{array}{l}32.9 \text { (TS) } \\
(30.0-36.0) \\
66.5 \\
(60.4-72.2)\end{array}$ & $\begin{array}{l}4.2 \\
(3.1-5.6) \\
10.4 \\
(6.9-14.7)\end{array}$ & $\begin{array}{l}3.7 \\
(2.6-5.0) \\
8.0 \\
(5.1-11.8)\end{array}$ & Prevalent & per 100py & & $\begin{array}{l}170.2 \text { (TS) } \\
(156.7-184.4) \\
114.7 \\
(103.3-129.1)\end{array}$ & $\begin{array}{l}168.2 \\
(130.0-213.9) \\
70.3 \\
(47.1-101.0)\end{array}$ & $\begin{array}{l}178.3 \\
(125.6-245.8) \\
122.8 \\
(83.4-174.3)\end{array}$ \\
\hline Gray [18] & $\begin{array}{l}\text { HIV-ve I, 15-49 } \\
\text { HIV-ve C, 15-49 }\end{array}$ & per 100py & - & $\begin{array}{l}19.7 \\
(15.3-24.9) \\
29.4 \\
(24.6-34.9)\end{array}$ & $\begin{array}{l}3.7 \\
(1.0-6.3) \\
4.9 \\
(3.1-7.6)\end{array}$ & $\begin{array}{l}1.7 \\
(0.6-3.7) \\
5.6 \\
(3.6-8.4)\end{array}$ & Prevalent & per 100py & & & $\begin{array}{l}190.9 \\
(118.2-291.8) \\
167.6 \\
(113.9-237.8)\end{array}$ & $\begin{array}{l}285.7 \\
(158.4-466.8) \\
157.9 \\
(88.4-260.4)\end{array}$ \\
\hline Serwadda [16] & $\begin{array}{l}\text { HIV + ve I, 15-49 } \\
\text { HIV + ve C, 15-49 }\end{array}$ & $\%$ at $24 \mathrm{~m}$ & - & $\begin{array}{l}42.0(\mathrm{TS}) \\
(31.1-55.5) \\
57.0 \\
(46.3-67.2)\end{array}$ & $\begin{array}{l}5.8 \\
(1.6-14.2) \\
14.9 \\
(7.7-25.0)\end{array}$ & $\begin{array}{l}4.3 \\
(0.9-12.0) \\
11.1 \\
(5.2-20.1)\end{array}$ & Prevalent & $\%$ at $24 \mathrm{~m}$ & & & $\begin{array}{l}66.7 \\
(34.9-90.1) \\
63.2 \\
(38.4-83.7)\end{array}$ & $\begin{array}{l}72.7 \\
(39.0-94.0) \\
91.7 \\
(61.5-99.8)\end{array}$ \\
\hline Kjaer [63] & Military, 18-29 & $\%$ at $6 \mathrm{~m}$ & $\begin{array}{l}13.8 \text { (NTS) } \\
(8.6-19.0)\end{array}$ & - & - & - & - & - & - & - & - & - \\
\hline Backes [17] & UnC, 17-24 & per 100py & $\begin{array}{l}\text { All: } 59.2 \\
\text { (NTS, first) } \\
(51.6-67.6) \\
\text { Glans: } 53.3 \\
\text { (46.1-60.5) } \\
\text { Shaft: } 25.9 \\
(22.3-30.0)\end{array}$ & $\begin{array}{l}\text { All: } 37.3 \\
\text { (32.0-43.2) } \\
\text { Glans: } 32.8 \\
(28.1-38.0) \\
\text { Shaft: } 15.7 \\
(13.1-18.7)\end{array}$ & $\begin{array}{l}\text { All: } 13.1 \\
\text { (10.7-15.6) } \\
\text { Glans: } 10.3 \\
(8.3-12.7) \\
\text { Shaft: } 5.3 \\
(3.8-7.0)\end{array}$ & $\begin{array}{l}\text { All: } 4.3 \\
\text { (3.1-5.9) } \\
\text { Glans: } 3.7 \\
\text { (2.6-5.3) } \\
\text { Shaft: } 1.8 \\
(1.1-2.9)\end{array}$ & - & - & - & - & - & - \\
\hline Mbulawa [22] & $\begin{array}{l}\text { All, } 18-66 \\
\text { HIV-ve, } 18-66 \\
\text { HIV + ve 18-66 }\end{array}$ & per 100py & $\begin{array}{l}66.8 \text { (TS, first) } \\
(52.3-83.0) \\
62.3 \\
(47.2-79.5) \\
96.5 \\
(57.1-163.2)\end{array}$ & $\begin{array}{l}35.7 \\
(28.1-45.4) \\
29.2 \\
(21.9-39.1) \\
72.9 \\
(47.2-112.8)\end{array}$ & $\begin{array}{l}3.8 \\
(2.2-6.5)\end{array}$ & $\begin{array}{l}4.0 \\
(2.4-6.8)\end{array}$ & Incident & per 100py & $\begin{array}{l}114.1 \text { (TS) } \\
(100.0-129.7) \\
128.2 \\
(115.4-141.9) \\
96.4 \\
(83.8-110.1)\end{array}$ & $\begin{array}{l}123.9 \\
(106.2-144.7)\end{array}$ & $\begin{array}{l}121.5 \\
(74.8-197.4)\end{array}$ & $\begin{array}{l}160.0 \\
(109.4-234.0)\end{array}$ \\
\hline $\begin{array}{l}\text { Darwich [20], } \\
\text { Videla [23] }\end{array}$ & $\begin{array}{l}\text { HIV + ve MSM, } \\
20->69 \\
\text { HIV + ve MSW, } \\
20->69\end{array}$ & per 100py & $\begin{array}{l}11.6 \text { (NTS, first) } \\
(8.8-14.9) \\
5.1 \\
(2.4-9.4)\end{array}$ & - & $\begin{array}{l}1.7 \\
(0.8-2.9) \\
1.4 \\
(0.4-3.7)\end{array}$ & $\begin{array}{l}0.4 \\
(0.1-1.1) \\
0.4 \\
(-)\end{array}$ & Prevalent & per 100py & $\begin{array}{l}27.1 \text { (NTS) } \\
(20.0-35.6) \\
23.6 \\
(14.8-35.6)\end{array}$ & - & $\begin{array}{l}29.4 \\
(15.1-51.4) \\
29.5 \\
(10.8-64.2)\end{array}$ & $\begin{array}{l}40.0 \\
(8.2-116.9) \\
14.6 \\
(-)\end{array}$ \\
\hline Wikström [21] & MSW, 18-54 & $\%$ at $3.5 \mathrm{~m}^{\mathrm{a}}$ & $\begin{array}{l}17.1(\mathrm{TS}) \\
(9.4-27.5)\end{array}$ & $\begin{array}{l}11.4 \\
(5.6-19.9)\end{array}$ & $\begin{array}{l}4.5 \\
(1.3-11.2)\end{array}$ & $\begin{array}{l}0 \\
(0-4.1)\end{array}$ & Prevalent & $\%$ at $3.5 \mathrm{~m}^{\mathrm{a}}-$ & $\begin{array}{l}41.7 \text { (TS) } \\
(15.2-72.3)\end{array}$ & $\begin{array}{l}42.9 \\
(9.9-81.6\end{array}$ & $\begin{array}{l}20 \\
(0.5-71.6)\end{array}$ & $\begin{array}{l}100 \\
\text { (1 case only) }\end{array}$ \\
\hline Giuliano [53] & HIV-ve, 18-70 & per 100py & $\begin{array}{l}46.1 \text { (NTS, first) } \\
(41.2-51.6)\end{array}$ & $\begin{array}{l}26.7 \\
(23.8-29.9)\end{array}$ & $\begin{array}{l}5.3 \\
(4.3-6.4)\end{array}$ & $\begin{array}{l}2.3 \\
(1.7-3.0)\end{array}$ & - & - & - & - & - & - \\
\hline
\end{tabular}


Table 2 Incidence and clearance of penile and other male genital HPV infections (Continued)

\section{Genital}

Giuliano [25]

glans, shaft,

scrotum)

Morales [26]

(scrotum, shaft,

balano-preputial

groove, urinary

meatus)

Partridge [29]

(glans \&urethral

meatus, shaft,

scrotum)

Lajous [27]

(shaft, coronal

sulcus, scrotum

urethral meatus)

Albero [28]

(shaft, coronal

sulcus, glans,

scrotum)

Kero [30]

(penis/urethra)
Men, 18-44

per 100py

46.1 (NTS, Total)

(41.8-61.2)

35.3 (NTS, first)

(27.4-44.9)

MSW, median 36 per 100py

14.8 (NTS, unclear) 6.7

(11.8-18.2)

MSW, 18-20

Male soldiers,

$16-40$

HIV-ve men

UnC, 18-70

HIV-ve men

Circ, 18-70

Male partners

of pregnant women, 20-52

\section{0}

18.6

62.4 (NTS, first)

(52.6-72.2)

(13.4-25.0)

5.8
$(3.4-9.2)$

$(0.2-2.9)$

Pr

$\begin{array}{lll}\text { valent } & \text { at } 6 \mathrm{~m} & 55.5 \\ \% \text { at } 12 \mathrm{~m} & 74.8 \\ \% \text { at } 18 \mathrm{~m} & 89.3\end{array}$

55.5 (NTS)

74.8

81.0

81.0
100

6.7
$(4.9-8.9)$

$(0.8-2.8)$

0.4

47.9

(38.6-58.0)

19.5

7.5

Abbreviations: MSM men who have sex with men, MSW men who have sex with women, py person-years, UnC Uncircumcised, Circ Circumcised, $m$ months, I Intervention (immediate circumcision), C Control (circumcision delayed for 24 months), TS type-specific incidence defined as at least one positive test for any HPV type or HR-HPV type not detected at baseline, NTS non-type-specific incidence defined as at least one positive test for any HPV type or HR-HPV type among those negative for any HPV DNA or any HR-HPV DNA at baseline

a Mean interval between visits $3.5 \mathrm{~m}$ : Results at visit 1 and 2 considered here 
years in HIV-ve men [19]. HPV types tending to clear the least rapidly were HPV-52 and 16 in circumcised men, 52 and 58 in uncircumcised men over a 2-year period [18], 52 and 16 in HIV + ve MSM and 18 and 51 in HIV + ve MSW up to 5 years of follow-up [20], depending on study and population. Age-specific clearance rates were highest in men aged $>30$ years in two studies $[19,22]$.

Time-to-clearance was 12.2 months for HPV-16 and 6.3 months for HPV-18 in HIV-ve men, versus 27.835.3 months for HPV-16 and 18 in HIV + ve men (Fig. 1).
HR-HPV persisted in $31 \%$ of HIV-ve men and $43 \%$ of HIV + ve men at 6 months, and in $25 \% / 28 \%$, respectively, at 12 months [24] (Table 3). Persistence of HR-HPV at 12 months was associated with higher HPV viral load at baseline in HIV + ve men [24].

\section{Cervical infections in comparable populations}

In South Africa, incidence and clearance of penile and cervical infections was assessed among 468 male-female couples aged 18-67 years (Table 4) [22]. Incidence and
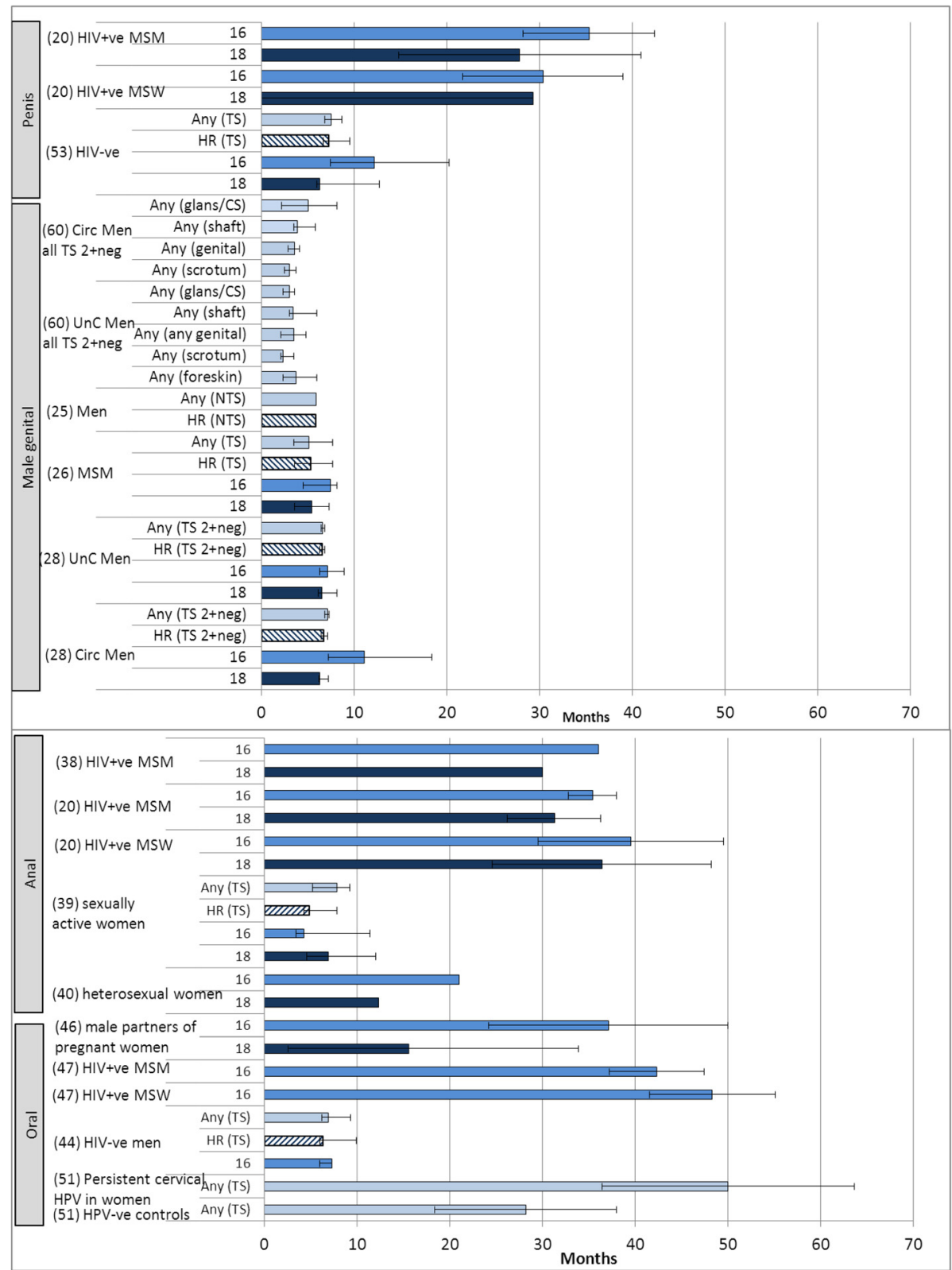

Fig. 1 Median* Time to clearance (months, $95 \%$ Cls) of HPV from non-cervical sites in men and women. Footnote: * mean reported for $[20,46,47,51]$. TS type-specific incidence defined as at least one positive test for any HPV type or HR-HPV type not detected at baseline; NTS non-type-specific incidence defined as at least one positive test for any HPV type or HR-HPV type among those negative for any HPV DNA or any HR-HPV DNA at baseline 
Table 3 Type-specific persistence of HPV infections

\begin{tabular}{|c|c|c|c|c|c|c|c|c|}
\hline \multirow[b]{2}{*}{ Site } & \multirow[b]{2}{*}{ Reference } & \multirow[b]{2}{*}{$\begin{array}{l}\text { Population, } \\
\text { age (years) }\end{array}$} & \multirow[b]{2}{*}{ Infection type } & \multirow[b]{2}{*}{$\begin{array}{l}\text { Time-frame } \\
\text { (months) }\end{array}$} & \multicolumn{4}{|c|}{$\%(95 \% \mathrm{Cl})$ persistent at end of time frame } \\
\hline & & & & & Any HPV & $\mathrm{HR}$ & 16 & 18 \\
\hline \multirow[t]{2}{*}{ Penile } & $\begin{array}{l}\text { Wikström; } \\
\text { Sweden [21] }\end{array}$ & MSW, 18-54 & $\begin{array}{l}\text { Prevalent/ } \\
\text { Incident }\end{array}$ & 3.5 & $50(23-77)$ & $57(18-90)$ & 75 (19-99) & $0(0-98)$ \\
\hline & $\begin{array}{l}\text { Grabowski; } \\
\text { Uganda [24] }\end{array}$ & $\begin{array}{l}\text { HIV- men, } \\
15-49 \\
\text { HIV+ men, } \\
15-49\end{array}$ & $\begin{array}{l}\text { Prevalent } \\
\text { Prevalent }\end{array}$ & $\begin{array}{l}6 \\
12 \\
24 \\
6 \\
12 \\
24\end{array}$ & & $\begin{array}{l}31(27-37) \\
25(20-30) \\
12(9-16) \\
43(37-48) \\
28(23-34) \\
29(25-34)\end{array}$ & & \\
\hline Anal & $\begin{array}{l}\text { Nyitray; Brazil, } \\
\text { Mexico, } \\
\text { USA [35] }\end{array}$ & $\begin{array}{l}\text { MSM, 18-70 } \\
\text { MSW, 18-70 }\end{array}$ & $\begin{array}{l}\text { Prevalent } \\
\text { Prevalent }\end{array}$ & $\begin{array}{l}6 \\
6\end{array}$ & $\begin{array}{l}59(48-70) \\
32(24-41)\end{array}$ & $\begin{array}{l}51(36-66) \\
24(14-37)\end{array}$ & $\begin{array}{l}73(39-94) \\
0(0-16)\end{array}$ & $\begin{array}{l}38(9-76) \\
0(0-84)\end{array}$ \\
\hline Male Genital & $\begin{array}{l}\text { Lajous; } \\
\text { Mexico [27] }\end{array}$ & $\begin{array}{l}\text { Male soldiers, } \\
16-40\end{array}$ & Prevalent & 12 & $29.4(21.6-38.2)$ & $31.0(22.1-41.0)$ & $31.3(11.0-58.7)$ & $0(0-33.6)$ \\
\hline \multirow[t]{2}{*}{ Oral } & $\begin{array}{l}\text { Pickard; } \\
\text { USA [49] }\end{array}$ & $\begin{array}{l}\text { Male/female } \\
\text { students, 18-30 }\end{array}$ & Prevalent & 3 & $38(19-59)$ & $42(15-72)$ & $0(0-98)$ & \\
\hline & $\begin{array}{l}\text { Kurose; } \\
\text { Japan [52] }\end{array}$ & $\begin{array}{l}\text { Male/female } \\
\text { volunteers, 3-85 }\end{array}$ & Prevalent & 30 & $50(9-93)$ & $0(0-98)$ & $0(0-98)$ & - \\
\hline
\end{tabular}

Abbreviations: MSM men who have sex with men, MSW men who have sex with women

clearance rates were significantly higher among men compared with women (incidence of any HPV 66.8 versus 40.7/100 person-years; clearance of any HPV 114.1 versus $80.3 / 100$ person-years). In regression analyses adjusting for HPV type, incidence rates remained higher in men when stratified by age-group and HIV status; clearance rates remained higher in men when stratified by age-group and among HIV + ve individuals (Table 4). HIV status was the only significant predictor of clearance in men and women, whereas in multivariate analyses, incidence was significantly predicted by HIV status and partner infection with same HPV type in men and women, and age at first sex and hormonal contraceptive use in women only.

\section{Male genital HPV infections}

Male genital sites included samples from the scrotum, coronal sulcus or combined samples of both penile and non-penile sites (Table 2).

\section{Incidence}

Incidence of any HPV ranged from 14.8 to 50.51/100 person-years in adult men and military men [25-28]. Incidence in university students (median follow-up of 12.9 months) was $62.4 \%$ [29]. Incidence in male partners of pregnant women was $32.3 \%$ over 7 years after the initial assessment [30]. HPV-16 was the most frequently identified type in all studies, followed by 52 and 58 [25-27, 29], or 51 and 59 [28].

Two studies reported on age-specific incidences of male genital infections, of which one was restricted to
18 or 19 year olds [29]. In an adult population the highest incidence was observed in the oldest age group studied (41-44 years) for any HPV infection (55.6/100 person-years), but the highest incidence of HR-HPV was in 26-30 year-olds (33.1/100 person-years) [31].

\section{Clearance and persistence}

Median time-to-clearance of any HPV male genital infection was 3.5-7.1 months (Fig. 1). Time-to-clearance of HPV-16 was 7.1-11.1 months, and 5.4-6.5 months for HPV-18.

The median duration of incident male genital HPV infections ranged between 5.1 and 7.1 months [25, 26, 28]. The duration of HPV-16 and/or 18 infection ranged between 5.4 and 11.1 months $[25,26,28]$. In the United States (US), HR-HPV genital infections persisted in $42 \%$ of men at 6 months, $19 \%$ at 12 months and had cleared in all men at 18 months [25] (Table 2).

\section{Cervical infections in comparable populations}

Incidence of male genital and cervical infections was assessed in two similar but separate cohorts of men and women from Arizona (Table 4) [25, 32]. Incidence calculated based on the first acquired infection with any HPV was similar in men and women (35.3/100 person-years [95 \%CI 27.4-44.9] versus 35.3/100 person-years [95\% CI 24.7-48.8]), but the cumulative incidence of infection by 12 months was substantially higher in men than in women (41\% versus $29 \%$ [95 \% CI 22-36] for any HPV; $32 \%$ versus $19 \%$ [95 \% CI 13-25) for HR-HPV). Median time-to-clearance for penile compared with cervical 
Table 4 Comparison of non-cervical HPV infections and cervical HPV infections in same or similar study populations

\begin{tabular}{|c|c|c|c|c|c|c|c|c|c|c|c|c|}
\hline \multirow[t]{2}{*}{ Ref, site } & \multirow[t]{2}{*}{ Population, age (years) } & \multicolumn{5}{|c|}{ Incidence (95 \% Cl) } & \multicolumn{6}{|c|}{ Clearance $(95 \% \mathrm{Cl})$} \\
\hline & & Unit & Any & $\mathrm{HR}$ & 16 & 18 & Inf. type & Unit & Any & $\mathrm{HR}$ & 16 & 18 \\
\hline \multirow[t]{2}{*}{$\begin{array}{l}\text { Darwich; Spain; } \\
\text { Penile [20] }\end{array}$} & $\begin{array}{l}\text { HIV + ve MSM, } \\
20->69\end{array}$ & Per 100py & - & - & $\begin{array}{l}1.7 \\
(0.8-2.9)\end{array}$ & $\begin{array}{l}0.4 \\
(0.1-1.1)\end{array}$ & Prev & Per 100py & - & - & $\begin{array}{l}29.4 \\
(15.1-51.4)\end{array}$ & $\begin{array}{l}40.0 \\
(8.2-116.9)\end{array}$ \\
\hline & $\begin{array}{l}\text { HIV + ve MSW, } \\
20->69\end{array}$ & Per 100py & - & - & $\begin{array}{l}1.4 \\
(0.4-3.7)\end{array}$ & $0.3(\mathrm{ND})$ & Prev & Per 100py & - & - & $\begin{array}{l}29.5 \\
(10.8-64.2)\end{array}$ & $\begin{array}{l}14.6 \\
(-)\end{array}$ \\
\hline $\begin{array}{l}\text { Videla; Spain; } \\
\text { Cervical [64] }\end{array}$ & $\begin{array}{l}\text { HIV + ve women, } \\
20-64\end{array}$ & $\%$ at $36 \mathrm{~m}$ & - & - & $\begin{array}{l}9 \\
(3.4-18.5)\end{array}$ & $\begin{array}{l}7 \\
(2.6-14.6)\end{array}$ & Prev & $\%$ at $36 \mathrm{~m}$ & - & - & $\begin{array}{l}46 \\
(26.6-66.6)\end{array}$ & $\begin{array}{l}29 \\
(3.7-71.0)\end{array}$ \\
\hline
\end{tabular}

Comment: Study period 1999-2003 for women v. 2005-2009 for men

\begin{tabular}{|c|c|c|c|c|c|c|c|c|c|c|c|c|}
\hline $\begin{array}{l}\text { Mbulawa; South Africa; } \\
\text { Penile [22] }\end{array}$ & $\begin{array}{l}\text { HIV + ve/-ve Men in a } \\
\text { relationship, 19-67 }\end{array}$ & Per 100py & $\begin{array}{l}66.8 \\
(T S, \text { first }) \\
(52.3-83.0)\end{array}$ & $\begin{array}{l}35.7 \\
(28.1-45.4)\end{array}$ & $\begin{array}{l}3.8 \\
(2.2-6.5)\end{array}$ & $\begin{array}{l}4.0 \\
(2.4-6.8)\end{array}$ & Prev/Inc & Per 100py & $\begin{array}{l}114.1 \text { (TS) } \\
(100-130.7)\end{array}$ & $\begin{array}{l}124.9 \\
(106.2-144.7)\end{array}$ & $\begin{array}{l}121.5 \\
(74.8-197.4)\end{array}$ & $\begin{array}{l}160.0 \\
(109.4-234.0)\end{array}$ \\
\hline Dulawa; South Africa; & HIV + ve/-ve Women in a & Per 100py & 40.7 (TS) & 18.7 & 2.4 & 1.4 & Prev/Inc & Per 100py & 80.3 (TS) & 74.7 & 38.9 & 127.9 \\
\hline
\end{tabular}

Cervical [22] relationship, 18-66 $\quad(31.7-52.2) \quad(13.8-25.7) \quad(1.3-4.7) \quad(0.6-3.3)$

Comment: Incidence and clearance significantly higher in men v. women. In type- adjusted analyses, incidence remained higher in men by age group and HIV status; clearance remained higher in men by age group and HIV + ve (96.4 versus 66.0/100 person-years), but not HIV-ve (128.1 versus 132.1/100 person-years)

\begin{tabular}{|c|c|c|c|c|c|c|}
\hline $\begin{array}{l}\text { Giuliano; USA (Arizona); } \\
\text { Male genital [25] }\end{array}$ & Adult men, 18-44 & Per 100py & $\begin{array}{l}46.1 \\
\text { (NTS, total) } \\
(41.8-61.2) \\
35.3 \\
\text { (NTS, first) } \\
\text { (27.4-44.9) }\end{array}$ & $\begin{array}{l}25.0) \\
(19.2-31.9) \\
18.6) \\
(13.4-25.0)\end{array}$ & $\begin{array}{l}5.3 \\
(3.4-9.2)\end{array}$ & $\begin{array}{l}1.0 \\
(0.2-2.9)\end{array}$ \\
\hline $\begin{array}{l}\text { Giuliano; USA (Arizona); } \\
\text { Cervical [32] }\end{array}$ & $\begin{array}{l}\text { Women attending } \\
\text { gynecology clinic,18-35 }\end{array}$ & Per 100py & $\begin{array}{l}35.3 \\
\text { (NTS, first) } \\
(24.7-48.8)\end{array}$ & - & $\begin{array}{l}7.1 \\
(3.7-12.1)\end{array}$ & $\begin{array}{l}1.0 \\
(0.1-3.5)\end{array}$ \\
\hline
\end{tabular}

Comment: Study period 2003-2005 for men v. 1996-1999 for women; follow-up at 6, 12, and 24 months for men v. 4 and 10 months for women. Med time to clearance anal v. cervical: 5.9 m (5.7-6.1) v. $9 \mathrm{~m}$ for any HPV; $5.8 \mathrm{~m}$ (5.5-6.1) v. $8.5 \mathrm{~m}$ for HR-HPV. PGMY09/11 assay used to detect types 61, 62, 64, 67, 69, 72, 81, IS39, and CP6108 in anal but not cervical samples

\begin{tabular}{|c|c|c|c|c|c|c|c|c|c|c|c|c|}
\hline $\begin{array}{l}\text { Goodman; USA (Hawaii); } \\
\text { Anal }[36,39]\end{array}$ & $\begin{array}{l}\text { Sexually active women, } \\
18-85\end{array}$ & Per 100py & $\begin{array}{l}56.3 \\
\text { (NTS, first) } \\
\text { (48.5-65.0) }\end{array}$ & $\begin{array}{l}23.4 \\
(19.2-28.3)\end{array}$ & $\begin{array}{l}3.0 \\
(1.8-4.8)\end{array}$ & $\begin{array}{l}2.3 \\
(1.2-3.9)\end{array}$ & Inc & Per 100py & $\begin{array}{l}89.3 \text { (TS) } \\
(67.8-115.4)\end{array}$ & $\begin{array}{l}110.0 \\
(83.3-142.4)\end{array}$ & $\begin{array}{l}141.2 \\
(73.0-246.7)\end{array}$ & $\begin{array}{l}130.0 \\
(47.6-282.8)\end{array}$ \\
\hline $\begin{array}{l}\text { Goodman; USA (Hawaii); } \\
\text { Cervical [41] }\end{array}$ & $\begin{array}{l}\text { Sexually active women, } \\
18-85\end{array}$ & Per 100py & $\begin{array}{l}15.6 \\
\text { (NTS, first) } \\
(12.9-18.7)\end{array}$ & $\begin{array}{l}11.1 \\
(9.0-13.6)\end{array}$ & $\begin{array}{l}2.1 \\
(1.4-3.2)\end{array}$ & $\begin{array}{l}0.6 \\
(0.2-1.2)\end{array}$ & Inc & Per 100py & $\begin{array}{l}85.1 \text { (TS) } \\
(64.2-110.4)\end{array}$ & $\begin{array}{l}92.0 \\
(67.8-122.0)\end{array}$ & $\begin{array}{l}86.4 \\
(41.4-158.9)\end{array}$ & $\begin{array}{l}69.7 \\
(14.4-203.6)\end{array}$ \\
\hline
\end{tabular}

Comment: 431 of 972 women in cervical analysis as per availability of anal sample, complete questionnaire data, and valid HPV testing results. Med time to clearance anal v. cervical: 7.8 m (5.2-9.2) v. $8.5 \mathrm{~m}$ (5.8-12.6) for any HPV; $4.9 \mathrm{~m}$ (4.3-8.0) v. $8.0 \mathrm{~m}$ (5.5-11.8) for HR HPV; 4.3 m (3.4-11.4) v. 9.5 m (4.4-23.8) for HPV16; 6.9 (4.6-12.0) v. 14.0 (4.8-ND) for HPV18
Moscicki:USA; (California);
Sexually active women
Anal [40]
$13-21$
Prev $\%$ at $12 \mathrm{~m}$ -
Moscicki;USA (California); Sexually active women, 13-22
Prev \% at $12 \mathrm{~m}$ -
$(19.0-41.7)$ 
Table 4 Comparison of non-cervical HPV infections and cervical HPV infections in same or similar study populations (Continued)

Comment: Anal and cervical analyses conducted on two partially overlapping cohorts of 75 and 531 women, respectively. Clearance defined as time to first of 2 consecutive negative results; data for any HPV not presented as cervical assay detected only 5 of 19 low-risk types detected by anal infection assay. HPV types 68, 69, 73, and 82 defined as HR types in analysis of anal but not cervical infection; $35 \%$ clearance at $12 \mathrm{~m}$ for cervical infections estimated from provided Kaplan-Meier curves

Vulvovaginal

Female university students, 100py

Winer; USA

Female university students, 100py

Cervical [43]

$$
\text { 18-20 }
$$

(NTS, first)

(13.7-18.6)

12.7

(NTS, first)

Comment: Separate vulvovaginal and cervical swabs collected from 444 initially HPV-DNA-negative women. $35.4 \%$ of infections were present at both sites

\begin{tabular}{|c|c|c|c|c|c|c|c|c|}
\hline $\begin{array}{l}\text { Kero, Finland, } \\
\text { Male Genital [30] }\end{array}$ & $\begin{array}{l}\text { Partners of pregnant } \\
\text { women, } 20-52\end{array}$ & $\%$ at 7 years & $\begin{array}{l}32.3 \text { (NTS) } \\
(16.7-51.4)\end{array}$ & - & - & Prev & $\%$ at 7 years & $\begin{array}{l}90 \text { (TS) } \\
(55.5-99.7)\end{array}$ \\
\hline $\begin{array}{l}\text { Kero; Finland; } \\
\text { Oral [30] }\end{array}$ & Pregnant women, 19-46 & $\%$ at 7 years & $\begin{array}{l}14.0 \text { (NTS) } \\
(5.3-27.9)\end{array}$ & - & - & Prev & $\%$ at 7 years & $\begin{array}{l}100 \text { (TS) } \\
(29.2-100)\end{array}$ \\
\hline Kero; Finland; & Pregnant women, 19-46 & $\%$ at 7 years & 16.7 (NTS) & - & - & Prev & $\%$ at 7 years & 70 (TS) \\
\hline
\end{tabular}

Cervical [30] (6.4-32.8) (34.8-93.3)

Comment: Analysis based on only 46 men and 46 women returning for follow-up at 84 months. At year 7 the same infecting HPV genotype was detected in $11 \%$ (95 \% Cl 0.3-48.2) and $30 \%$ (6.7-65.2) of mean and women, respectively. HPV status during interval visits was not taken into account.

Note: For male versus female comparison only date for penile/male genital infections versus cervical infections are presented

Abbreviations: $m$ months, - data not provided, TS type-specific incidence defined as at least one positive test for any HPV type or HR-HPV type not detected at baseline, NTS non-type-specific incidence defined as at

least one positive test for any HPV type or HR-HPV type among those negative for any HPV DNA or any HR-HPV DNA at baseline 
infections was 5.9 months (95\% CI 5.7-6.1) versus approximately 9 months for any HPV, and 5.8 months $(95 \%$ CI 5.5-6.1) versus 8.5 months for HR-HPV. In Finland, where male genital and cervical infection data were collected at baseline and 7 years later among 46 pregnant women and their male partners, incidence and clearance of any HPV was also higher among men versus women: 32.3 \% (95 \% CI 16.7-51.4) versus 16.7 \% (95 \% CI 6.432.8 ) and $90 \%$ (95 \% CI 55.5-99.7) versus $70.0 \%$ (95 \% CI 34.8-93.3) (Table 4). Among men and women with genital HPV infections at baseline, the same infecting HPV genotype was present at the 7-year follow-up in $11 \%$ (95\% CI $0.3-48.2)$ and $30 \%(6.7-65.2)$, respectively [30].

\section{Anal HPV infections}

Ten articles reported on anal HPV infections: seven reported infections in men and four in women (Table 5).

\section{Incidence in men}

The incidence of anal HPV infection was higher in HIV + ve men (gender of partner not specified) and HIV-ve MSM (range 21.3-46.2/100 person-years), than in HIV + ve MSW (7.9/100 person-years) and MSW (HIV status not specified, 9.7/100 person-years). Among MSM, HPV16 and 18 were among the HPV types with the highest incidence, although depending on the study, the incidences of HPV-51, 52 or 59 were similar or higher [33-35].

\section{Incidence in women}

In women, anal HPV incidence ranged from 14-56.3/100 person-years. HPV- 16 and 52 were the most frequently infecting types [36]. The incidence of any and HR anal HPV infection in 12-18 year olds was higher in men than women (HIV-ve and HIV + ve), although the 95\%CIs overlapped (Table 5) [37]. By contrast, in another study in women [36] the incidence (per 100 person-years) of any anal HPV infection was higher (56.3) than in all studies reported in male populations (range 7.9-46.2) (Table 5). In this study the incidence (per 100 person-years) of HRHPV infection in women (23.4) was higher than that reported in MSW (4.4) and within the range observed in HIV + ve men or MSM (13.3-36.7).

A regression analysis conducted in a US study of sexually active women noted a statistically significant inverse relationship between acquisition of a new HR-HPV anal infection and age; with a $57 \%$ (95 \% CI 19-77) lower risk of acquisition among older women $(\geq 45$ years of age at baseline) than among younger women $(<25$ years at baseline) [36].

\section{Clearance and persistence in men and women}

Seven articles reported on the clearance rate of anal infections, of which five were in men who were HIV + ve and/ or MSM, and two were in women (Table 5). The clearance rate of prevalent anal HPV infections in men varied between 14.6 and 66.7/100 person-years for specific HPV types. Amongst HR-HPV, the clearance rate was lowest for HPV-16 in 5/6 populations [20, 33, 35, 38]. For HPV16 and 18 the time-to-clearance ranged between 30 and 39.5 months (Fig. 1).

In women, the clearance rate of incident anal HPV infections was $89.3 / 100$ person-years, with a median timeto-clearance of 7.8 months [39] (Fig. 1). In another study, $56.5 \%$ of women cleared any HR-HPV infection by year 3 [40]. In the single study that reported clearance of prevalent infections, HPV clearance in women appeared to be within the range reported in studies in men (Table 5).

HR-HPV anal infections persisted at 6 months in $51.0 \%$ of MSW and in $24.2 \%$ of MSM who were HRHPV positive at baseline [35] (Table 3).

\section{Cervical infections in comparable populations}

In Hawaii, anal and cervical samples were collected in sexually active women aged $18-85$ years every 4 months for an average of approximately 1.3 years [36, 39, 41]. Incidence (per 100 person-years) of anal versus cervical infections was more than three-fold higher for any HPV (56.3 [95 \% CI 48.5-65.0] versus 15.6 [95\% CI 12.9-18.7]) and more than two-fold higher for HR-HPV (23.4 [95 \% CI 19.2-28.3] versus 11.1 [95\% CI 9.0-13.6]); differences were similar for HPV-16 and 18 infections but not statistically significant (Table 4). Baseline cervical HPV status was not a significant risk factor for incidence of anal HPV infection. In contrast, clearance (per 100 person-years) did not significantly differ between anal versus cervical infections with either any HPV (89.3 [95 \% CI 67.8-115.4] versus 85.1 [95 \% CI 64.2110.4], respectively) or with HR-HPV (109.9 [95 \% CI 83.3142.4] versus 92.0 [95 \% CI 67.8-122.0], respectively).

In two partially overlapping cohorts of sexually active teenager and young adults in the US, clearance by 12 months was also similar for anal versus cervical infections (29.7 \% [95 \% CI 19.0-41.7] and approximately $35 \%$, respectively) [40, 42]. In the Hawaii cohort, there was some indication of higher clearance of anal than cervical HPV-16 and 18 infections, with more than twofold differences in the median duration of infection (4.3 versus 9.8 months, and 6.9 versus 14 months, respectively), but CIs overlapped substantially (data not shown) $[36,39,41]$.

\section{Vaginal HPV infections}

We identified one study of vulvovaginal HPV infections that employed physician-collected swabs [43]. The incidence rate for vulvovaginal HPV infections among university students was 16.0 per 100 person-years (95\% CI 13.7-18.6) compared with 12.7 per 100 person-years (95\% CI 10.8-14.9) for cervical infections (Table 4). No information on clearance or persistence was available. 
Table 5 Incidence and clearance of anal HPV infections

\begin{tabular}{|c|c|c|c|c|c|c|c|c|c|c|c|}
\hline \multirow[t]{2}{*}{ Author (ref) } & \multirow[t]{2}{*}{ Population, age (yrs) } & \multicolumn{4}{|c|}{ Incidence (per 100py) } & \multicolumn{6}{|l|}{ Clearance } \\
\hline & & Any HPV & $\mathrm{HR}$ & 16 & 18 & Type of Infection & Unit of clearance & Any HPV & $\mathrm{HR}$ & 16 & 18 \\
\hline \multicolumn{12}{|l|}{ Males } \\
\hline $\begin{array}{l}\text { de Pokomandy } \\
\text { [38] }\end{array}$ & $\begin{array}{l}\text { HIV + ve MSM, } \\
21-66\end{array}$ & - & - & $\begin{array}{l}13.0 \\
(9.6-17.6)\end{array}$ & $\begin{array}{l}5.3 \\
(3.5-8.0)\end{array}$ & Prevalent & Per 100py & & & $\begin{array}{l}14.6 \\
(10.2-21.2)\end{array}$ & $\begin{array}{l}24.5 \\
(16.9-35.4)\end{array}$ \\
\hline Nyitray [35] & $\begin{array}{l}\text { MSM, 18-70 } \\
\text { MSW, 18-70 }\end{array}$ & $\begin{array}{l}31.1 \\
(\mathrm{TS}) \\
(16.6-53.2) \\
9.7 \\
(7.2-12.8)\end{array}$ & $\begin{array}{l}25.4 \\
(14.5-41.2) \\
4.4 \\
(2.9-6.6)\end{array}$ & $\begin{array}{l}5.8 \\
(1.9-13.4) \\
0.8 \\
(0.2-2.0)\end{array}$ & $\begin{array}{l}4.6 \\
(1.2-11.6) \\
1.1 \\
(0.4-2.3)\end{array}$ & Prevalent & $\%$ at $6 \mathrm{~m}-$ & $\begin{array}{l}57.1 \text { (TS) } \\
(50.1-63.8) \\
70.9 \\
(63.5-77.5)\end{array}$ & $\begin{array}{l}55.8 \\
(44.1-67.2) \\
77.5 \\
(66.0-86.5)\end{array}$ & $\begin{array}{l}27.2 \\
(6.0-61.0) \\
100 \\
(83.9-100)\end{array}$ & $\begin{array}{l}62.5 \\
(24.5-91.5) \\
100 \\
(15.8-100)\end{array}$ \\
\hline Videla [23] & $\begin{array}{l}\text { HIV + ve MSM, } \\
20->69 \\
\text { HIV + ve MSW, } \\
20->69\end{array}$ & $\begin{array}{l}32.4 \\
\text { (NTS) } \\
(23.3-43.7) \\
7.9 \\
(4.9-15.7)\end{array}$ & - & - & - & Prevalent & Per 100py & $\begin{array}{l}12.6 \text { (NTS) } \\
(10.2-15.2) \\
18.4 \\
(12.2-26.5)\end{array}$ & - & - & - \\
\hline Darwich [20] & $\begin{array}{l}\text { HIV + ve MSM, } \\
20->69 \\
\text { HIV + ve MSW, } \\
20->69\end{array}$ & - & - & $\begin{array}{l}7.1 \\
(5.5-9.5) \\
5.3 \\
(3.0-8.6)\end{array}$ & $\begin{array}{l}3.6 \\
(3.5-5.2) \\
0.4 \\
(0.1-1.8)\end{array}$ & Prevalent & Per 100py & - & & $\begin{array}{l}22.4 \\
(17.6-28.1) \\
18.6 \\
(7.4-38.2)\end{array}$ & $\begin{array}{l}31.0 \\
(20.3-45.4) \\
30.0 \\
(7.8-76.8)\end{array}$ \\
\hline Mullins [37] & $\begin{array}{l}\text { HIV-ve, } 12-18 \\
\text { HIV + ve, 12-18 }\end{array}$ & $\begin{array}{l}24 \\
\text { (NTS, first) } \\
(11-52) \\
40 \\
(27-61)\end{array}$ & $\begin{array}{l}11 \\
(3.4-33) \\
27 \\
(17-44)\end{array}$ & - & - & - & - & - & - & - & - \\
\hline Glick [33] & MSM, 16-30 & $\begin{array}{l}46.2 \\
\text { (NTS, unclear) } \\
(45.6-46.9)\end{array}$ & $\begin{array}{l}36.7 \\
(36.2-37.3)\end{array}$ & $\begin{array}{l}12.4 \\
(12.1-12.6)\end{array}$ & $\begin{array}{l}5.5 \\
(5.3-5.6)\end{array}$ & Prevalent & $\%$ at 1 year & $\begin{array}{l}81.3 \text { (TS) } \\
(67.4-91.1)\end{array}$ & $\begin{array}{l}66.7 \\
(49.0-81.4)\end{array}$ & $\begin{array}{l}50.0 \\
(26.0-74.0)\end{array}$ & $\begin{array}{l}66.7 \\
(22.3-95.7)\end{array}$ \\
\hline Hernandez [34] & $\begin{array}{l}\text { HIV + ve MSM, } \\
\text { mean } 45\end{array}$ & $\begin{array}{l}21.3 \text { (TS, total) } \\
(17.7-25.4)\end{array}$ & $\begin{array}{l}13.3 \\
(10.5-16.6)\end{array}$ & $\begin{array}{l}3.5 \\
(1.8-6.1)\end{array}$ & $\begin{array}{l}3.7 \\
(2.1-6.1)\end{array}$ & - & - & - & - & - & - \\
\hline \multicolumn{12}{|l|}{ Females } \\
\hline $\begin{array}{l}\text { Goodman [36], } \\
\text { Shvetsov [39] }\end{array}$ & $\begin{array}{l}\text { Sexually active } \\
\text { women, 18-44 }\end{array}$ & $\begin{array}{l}56.3 \\
\text { (NTS, first) } \\
(48.5-65.0)\end{array}$ & $\begin{array}{l}23.4 \\
(19.2-28.3)\end{array}$ & $\begin{array}{l}3.0 \\
(1.8-4.8)\end{array}$ & $\begin{array}{l}2.3 \\
(1.2-3.9)\end{array}$ & Incident & Per 100py & $\begin{array}{l}89.3 \text { (TS) } \\
(67.8-115.4)\end{array}$ & $\begin{array}{l}109.9 \\
(83.3-142.4)\end{array}$ & $\begin{array}{l}141.2 \\
(73.0-246.7)\end{array}$ & $\begin{array}{l}130.0 \\
(47.6-282.8)\end{array}$ \\
\hline Moscicki [40] & $\begin{array}{l}\text { Sexually active } \\
\text { women, 13-21 }\end{array}$ & - & - & - & - & Prevalent & $\%$ at $12 \mathrm{~m}$ & - & $\begin{array}{l}29.7 \text { (TS 2+ neg) } \\
(19.0-41.7)\end{array}$ & $\begin{array}{l}45.0 \\
(27.2-62.1)\end{array}$ & $\begin{array}{l}55.6 \\
(21.2-86.3)\end{array}$ \\
\hline Mullins [37] & $\begin{array}{l}\text { HIV-ve women, } \\
12-18 \\
\text { HIV + ve women, } \\
12-18\end{array}$ & $\begin{array}{l}14 \\
\text { (NTS,first) } \\
(9.2-22) \\
30 \\
(24-38)\end{array}$ & $\begin{array}{l}5.3 \\
(2.6-11) \\
12 \\
(8.4-16)\end{array}$ & - & - & - & - & - & - & - & - \\
\hline
\end{tabular}

Abbreviations: MSM men who have sex with men, MSW men who have sex with women, py person-years, TS type-specific incidence defined as at least one positive test for any HPV type or HR-HPV type not detected at baseline, NTS non-type-specific incidence defined as at least one positive test for any HPV type or HR-HPV type among those negative for any HPV DNA or any HR-HPV DNA at baseline 


\section{Oral HPV infections}

Eleven articles (considering six cohorts) reported on oral HPV infections: eight in men and five in women (Table 6).

\section{Incidence in men}

The incidence of oral HPV infection was 5.7-6.1/100 person-years in HIV + ve men and 6.7/100 person-years in HIV-ve men $[23,44]$. In another study conducted in university students, oral HPV incidence over 1 year was $12.3 \%$ [45]. Finally, in the Finnish cohort, oral HPV infection was reported in 14.3-69.2 \% of male partners of pregnant women 7 years after baseline. The variability in results was due to the varying sampling timetable [30, 46]. In studies reporting type-specific incidence, HPV-16 was the most frequently identified type [44, 45, 47, 48].

Two studies reported age-specific incidence rates of oral infections in men. In one study in university students the age-specific incidence was higher in the 21-24 years age-group than in the 18-20 years age-group [45]. In HIV-ve men there was no increased risk of incident HR-HPV oral infection across age groups (range 18-73 years) [44].

\section{Incidence in women}

The incidence of oral HPV infections in HIV-ve women was $6.8-20.4 / 100$ person-years versus $39.6 / 100$ personyears in HIV + ve women [15, 49]. In the Finnish cohort, oral infections were present in approximately $10 \%$ of men and women after 24 months follow-up, and in $14.3 \%$ of men and women after 7 years [30, 50]. Incidence rates in women appeared to be similar or higher than rates in men, depending on the study.

\section{Clearance and persistence in men and women}

Clearance rates of oral infections in men were reported in two study cohorts (Table 6). In HIV + ve men, the clearance rate of prevalent oral infections in MSM was 19.3/100 person-years, or $48.3 \%$ after two-year followup [23, 47]. Rates for HIV + ve MSW were 15.8/100 person-years and $34 \%$, respectively [23, 47]. The timeto-clearance of oral HR-HPV in HIV-ve men was 6.9 months. Time-to-clearance of oral HPV-16 infection was between 7.3 and 37.1 months in HIV-ve men, and was higher (42.3-48.3 months) in HIV + ve men (Fig. 1). In the Finnish cohort, approximately $5 \%$ of oral HRHPV infections in 19 HR-HPV-positive men had cleared by 24 months [50].

In US studies, clearance of any oral HPV in women was $60.6 \%$ at 3 months [49], and clearance at 6 months was $20.0 \%$ in HIV-ve women and $40.0 \%$ in HIV + ve women [15]. No oral HPV infections had cleared in women in the Finnish cohort at 24 months, whereas clearance at 7 years was $100 \%$ [30, 50]. Among women with persistent cervical HPV infection the time-to-clearance of oral HPV infection was 50.0 months, versus 28.2 months in women without cervical HPV infections (Fig. 1) [51].

Persistence of oral HPV infections was reported for three cohorts (Table 3). In 662 healthy volunteers in Japan, four had a prevalent infection and two had persistent infection with the same HPV-type after 2.5 years [52]. The persistence of prevalent HR-HPV infections in male and female university students was $42 \%$ after three months [49].

\section{Risk factors for non-cervical and oral HR-HPV infection}

Penile and/or male genital infections with HR-HPV were significantly associated with younger age in only one study [19], although three articles reported no significant association with age $[17,31,53]$. Other significant risk factors were anal intercourse, HPV status of the partner, number of partners (current or lifetime), and HIV infection $[17,19,22,26,31,53]$. Being uncircumcised was identified as a risk factor for penile HR-HPV infection in one study in HIV-ve men [19], but not in another [28].

An increased likelihood of clearance of HR-HPV penile infection was associated with an increased number of sex partners and circumcision (in HIV-ve men) $[19,31]$. One study reported increased clearance in men with self-reported urethral discharge [19], but other sexually-transmitted genital infections were not associated with clearance [19, 31].

Risk factors for anal HR-HPV infection in women were younger age, condom use (possibly due to using the same condom for vaginal and anal sex), higher lifetime number of partners $(\geq 6)$, and advanced HIV infection and smoking (in HIV + ve women) [36, 37]. There was decreased likelihood of clearance of anal HR-HPV infection in women with a 2-10-pack-year smoking history [39].

No risk factors were significantly associated (multivariate analyses) with anal HR-HPV infection in men.

Risk factors for persistence of oral HR-HPV were only available from one study: a history of smoking was significantly associated with longer persistence whereas a history of genital warts was associated with shorter persistence [46].

\section{Discussion}

The available data suggest a high incidence of noncervical HPV infections in men and women, with HPV16 most consistently ranking among the HR-HPV types with the highest incidence rates and the lowest clearance rates in all sites. Despite relatively few studies providing data for cervical and other genital infections in comparable populations, there appears to be a general pattern of both higher incidence and clearance of non-cervical versus cervical genital infections. Around one-half of the identified articles assessed HPV at male penile/other genital sites. There was a wide variation in HPV incidence reported at these sites, although the time-to- 
Table 6 Incidence and clearance of oral HPV infections

\begin{tabular}{|c|c|c|c|c|c|c|c|c|c|c|c|c|}
\hline \multirow[t]{2}{*}{ Author (ref) } & \multirow[t]{2}{*}{ Population, age (years) } & \multicolumn{5}{|c|}{ Incidence (95 \% Cl) } & \multicolumn{6}{|l|}{ Clearance } \\
\hline & & Unit & Any HPV & $\mathrm{HR}$ & 16 & 18 & Infection type & Unit of clearance & Any HPV & $\mathrm{HR}$ & 16 & 18 \\
\hline \multicolumn{13}{|l|}{ Males } \\
\hline Edelstein [45] & $\begin{array}{l}\text { Male students, } \\
\text { median } 20\end{array}$ & $\%$ at $1 y$ & $\begin{array}{l}12.3 \% \\
\text { (NTS, first) } \\
(7.0-21.3)\end{array}$ & - & $\begin{array}{l}0.8 \\
(0.1-5.7)\end{array}$ & $\begin{array}{l}2.7 \\
(0.7-10.2)\end{array}$ & - & - & - & - & - & - \\
\hline Kero [48] & $\begin{array}{l}\text { Male partners of } \\
\text { pregnant women, } \\
19-46\end{array}$ & per 100py & - & - & $\begin{array}{l}6.0 \\
(2.9-9.2)\end{array}$ & $\begin{array}{l}1.2 \\
(0.2-3.4)\end{array}$ & - & - & - & - & - & - \\
\hline Kero $[30]^{a}$ & $\begin{array}{l}\text { Male partners of } \\
\text { pregnant women, } \\
20-52\end{array}$ & $\%$ at 7 years & $\begin{array}{l}14.3 \% \\
\text { (NTS) } \\
\text { (4.8-30.3) }\end{array}$ & - & - & - & Prevalent & $\%$ at 7 years & $\begin{array}{l}100 \%(\text { (TS) } \\
(64.4-100)\end{array}$ & - & - & - \\
\hline Kero $[46]^{\mathrm{a}}$ & $\begin{array}{l}\text { Male partners of } \\
\text { pregnant women, } \\
20-52\end{array}$ & $\%$ at 7 years & $\begin{array}{l}69.2 \% \text { (TS) } \\
(59.5-77.2)\end{array}$ & - & - & - & Prevalent & per 100py & $\begin{array}{l}3.8 \text { (TS) } \\
(1.6-6.1)\end{array}$ & - & $\begin{array}{l}4.9 \\
(2.3-7.4)\end{array}$ & $\begin{array}{l}1.4 \\
(0-2.7)\end{array}$ \\
\hline Rintala [50] & $\begin{array}{l}\text { Male partners of } \\
\text { pregnant women, } \\
20-52\end{array}$ & $\%$ at $24 \mathrm{~m}$ & - & $\begin{array}{l}\sim 10 \% \\
\text { (NTS, first) }\end{array}$ & - & - & Prevalent & $\%$ at $24 \mathrm{~m}$ & - & $\sim 5 \%$ (NTS) & - & - \\
\hline Videla [23] & $\begin{array}{l}\text { HIV + ve MSM, } \\
20->69 \\
\text { HIV + ve MSW, } \\
20->69\end{array}$ & per 100py & $\begin{array}{l}6.1 \\
\text { (NTS, first) } \\
(4.2-8.4) \\
5.7 \\
(3.0-9.7)\end{array}$ & - & - & - & Prevalent & per 100py & $\begin{array}{l}19.3 \\
\text { (NTS) } \\
(12.8-27.7) \\
15.8 \\
(7.6-29.0)\end{array}$ & - & - & \\
\hline Darwich [47] & $\begin{array}{l}\text { HIV + ve MSM, } \\
20->69 \\
\text { HIV + ve MSW, } \\
20->69\end{array}$ & per 100py & - & - & $\begin{array}{l}1.0 \\
(0.4-2.0) \\
2.6 \\
(1.1-5.4)\end{array}$ & $\begin{array}{l}0.2 \\
(0-1.0) \\
0.7 \\
(0.1-2.4)\end{array}$ & Prevalent & $\%$ at $24 \mathrm{~m}$ & $\begin{array}{l}48.3 \% \text { (TS) } \\
(35.2-61.6) \\
34 \% \\
(17.9-54.3)\end{array}$ & - & $\begin{array}{l}22.7 \\
\text { per 100py } \\
(11.3-40.6)\end{array}$ & $\begin{array}{l}9.1 \\
\text { per 100py } \\
(1.8-26.5)\end{array}$ \\
\hline Kreimer [44] & $\begin{array}{l}\text { HIV-ve men, } \\
18-70\end{array}$ & per 100py & $\begin{array}{l}6.7 \\
\text { (NTS, first) } \\
(5.5-8.0)\end{array}$ & $\begin{array}{l}3.0 \\
(2.2-3.8)\end{array}$ & $\begin{array}{l}1.0 \\
(0.6-1.6)\end{array}$ & $\begin{array}{l}0.0 \\
(0-0.4)\end{array}$ & - & - & - & - & - & - \\
\hline \multicolumn{13}{|l|}{ Women } \\
\hline Pickard [49] & $\begin{array}{l}\text { Female students, } \\
18-30\end{array}$ & per 100py & $\begin{array}{l}6.8 \text { (TS) } \\
(3.7-9.8)\end{array}$ & - & - & - & Prevalent & $\%$ at $3 \mathrm{~m}$ & $\begin{array}{l}60.6 \\
(\mathrm{TS}) \\
(42.1-77.1)\end{array}$ & $\begin{array}{l}76.9 \\
(46.2-95.0)-\end{array}$ & $\begin{array}{l}100 \\
(15.8-100)-\end{array}$ & - \\
\hline D'Souza [15] & $\begin{array}{l}\text { HIV-ve women } \\
\text { HIV + ve women }\end{array}$ & per 100py & $\begin{array}{l}20.4 \text { (TS) } \\
(8.4-44.4) \\
39.6 \\
(25.2-57.6)\end{array}$ & - & - & - & Prevalent & $\%$ at $6 \mathrm{~m}$ & $\begin{array}{l}20.0 \\
\text { (NTS) } \\
(0.5-71.6) \\
40.0 \\
(22.7-59.4)\end{array}$ & - & - & - \\
\hline Kero [30] & $\begin{array}{l}\text { Pregnant women, } \\
19-46\end{array}$ & $\%$ at 7 years & $\begin{array}{l}14.3 \% \text { (NTS) } \\
(5.4-28.5)\end{array}$ & - & - & - & Prevalent & $\%$ at 7 years & $\begin{array}{l}100 \%(\text { TS) } \\
(29.2-100)\end{array}$ & - & - & - \\
\hline Rintala [50] & $\begin{array}{l}\text { Pregnant women, } \\
19-46\end{array}$ & $\%$ at $24 \mathrm{~m}$ & - & $\begin{array}{l}\sim 10 \% \\
\text { (NTS, first) }\end{array}$ & - & - & Prevalent & $\%$ at $24 \mathrm{~m}$ & - & $0 \%$ (NTS) & - & - \\
\hline
\end{tabular}


Table 6 Incidence and clearance of oral HPV infections (Continued)

\section{Men and women}

Male/female volunteers, -

Prevalent

$\%$ at $30 \mathrm{~m}$

50.0 (TS)

$(6.8-93.2)$

100

Abbreviations: MSM men who have sex with men, MSW men who have sex with women, py person-years, $y$ years, $m$ months, TS type-specific incidence defined as at least one positive test for any HPV type or HR-HPV

type not detected at baseline, NTS non-type-specific incidence defined as at least one positive test for any HPV type or HR-HPV type among those negative for any HPV DNA or any HR-HPV DNA at baseline

aStudy [30] considered a subset of men (46/131) included in [46]. Study [30] reported HPV status at baseline and at 7 years, whereas study [46] considered HPV status at baseline and all subsequent follow-up visits

(i.e., $2 \mathrm{~m}, 6 \mathrm{~m}, 24 \mathrm{~m}, 36 \mathrm{~m}$, and $7 \mathrm{y}$ ) 
clearance was comparable across all studies, with the exception of substantially longer time-to-clearance in HIV + ve men. HIV status, circumcision, number of sex partners and HPV status of the partner significantly influenced HR-HPV incidence, clearance and persistence rates at male anogenital sites in some, but not all studies. Some of these risk factors are the same as those identified for cervical HPV infection, for which the number of sex partners is the most important one [54].

Unlike cervical intraepithelial neoplasia, analintraepithelial neoplasia infrequently undergoes malignant transformation in immunocompetent individuals, but the risk of malignant transformation is much greater in HIV + ve individuals [55]. Corresponding with the higher incidence of anal cancer in MSM and HIV + ve men compared to the general population, we also found consistently higher incidences of anal HPV infection in these groups compared to MSW and HIV-ve men, respectively. There was a lack of similar comparison data for these risk groups for clearance. The primary exception was Nyitray et al. [35], which indicated potentially lower clearance rates for MSM versus MSW, but multivariate analyses directly comparing the two groups within the same model were not conducted.

The Hawaiian studies by Goodman et al. showed an incidence of anal HPV infection that was three-times higher than cervical HPV infection [36, 39, 41], although a recent review concluded that the prevalence of anal and cervical infections are similar in women, and that cervical HPV infection is a risk factor for anal HPV infection [56]. As prevalence is determined by the incidence rate multiplied by the average duration of infection, the similar prevalence between anal and cervical infections could be due the higher incidence of anal infections being offset by simultaneously higher clearance. The Hawaiian cohorts provided indication of higher clearance of anal infections, but despite this study having one of the largest, most carefully followed cohorts, there were limited samples sizes to assess clearance rates, especially for individual types. This was in part due to the fact that the analyses of clearance focused on infections that were acquired during the course of the study.

The incidence of oral HPV infection was within the same range in men and women, although the prevalence of oral HPV infection has been reported to be higher in men [57]. Our data were too limited to conclude whether there were differences in persistence or clearance rates between men and women. It is thought that the oncogenicity of HPV is similar in oral and cervical cancers, although numerous gaps remain in understanding the risk factors that influence progression from oral infection to oral neoplasia [57].

The factors that influence oncogenesis at non-cervical sites are not well understood, and appear to differ by site and between populations (including men versus women, immunosuppressed versus immunocompetent, circumcised versus uncircumcised) $[57,58]$. Although the incidence of HPV infection at non-cervical sites appears to be high, the increased capacity for clearance of HPV observed may contribute to the lower occurrence of these types of cancers. Overall, data describing the natural history of non-cervical cancers are lacking.

A potential limitation of our study is the non-inclusion of articles in which vaginal swabs were self-collected. The single study that we identified using physician-collected vaginal swabs may not be representative for this site as it was limited to university students. However, it was one of the highest quality studies, following more than 400 women aged 18-20 years old every 4 months for 10 years and prospectively collecting sexual behavior data. The studies on cervical infections were selected from specific non-systematic searches aimed to identify comparable populations with which to compare to non-cervical infections. Thus, the results may not be applicable beyond the study setting. Most studies were not properly designed for risk factor analysis and should be interpreted cautiously.

The studies we identified were substantially different in terms of their design, including follow-up times and sampling intervals which ranged from several months to 7 years, as well as the number of types and methods used for HPV genotyping. Although it is difficult to apply a standard grading to these studies, the highest quality studies tended to be those that followed cohorts of at least 200 subjects and conducted multiple follow-up visits $\leq 6$ months apart for at least one year. At least one such study provided data for each of the sampling sites: penile [17-19, 22, 24, 53], male genital $[25,26,28,31,53,59]$, anal $[34,36,38,39]$, vulvovaginal [43], oral [44].

The available published data are limited by the lack of age-specific estimates, and information on clearance/persistence of infection was frequently based on very small sample sizes, resulting in imprecise estimates. Few studies provided incidence or clearance data on oral or anal HPV infections in women versus men, and the relative burden of these infections in each sex is not clear. Specific estimates of incidence and clearance of HPV-16, which is involved in the majority of non-cervical cancers, and HPV18 which may also be important in some sites, as well as estimation of clearance rates at 6 and 12 months, could inform on potentially important endpoints for clinical trials of vaccine efficacy. Finally, because just a few countries contributed data to this review and given the important differences in study designs, outcomes and populations, it is important to note the limited generalizability of these results to other populations.

\section{Conclusions}

Cases of cervical cancer are projected to increase, emphasising the importance of ongoing efforts to provide access 
of effective HPV vaccines to all $[1,60]$. As yet, the implications of HPV vaccination for prevention of non-cervical cancers have not been fully explored [58]. Some countries have recommended HPV vaccination for young males on the basis that vaccination will prevent HPV-associated cancers in men, as well as theoretical benefits in preventing transmission of HPV to women [61, 62]. Programs targeting prevention of cervical HPV infection could have additional benefits on the non-cervical disease burden. This review suggests that there are parallels between the epidemiology of cervical and non-cervical HPV infections in terms of the incidence and distribution of HPV types, and of risk factors for HPV infection. However, these parallels may not be directly applicable to disease at noncervical sites. More detailed and extensive studies could provide useful information for estimating vaccine impact, the wider cost-benefit of HPV vaccination, and for guiding vaccination policy.

\section{Additional files}

Additional file 1: Search strings; Article screening; Data extraction; Identification of papers on cervical HPV infection in same/similar study populations; Quality control. (DOCX $139 \mathrm{~kb}$ )

Additional file 2: Table S1. Summary of HPV testing conducted in 38 articles included in the review. (XLSX $38 \mathrm{~kb}$ )

\section{Abbreviations}

$2+$ neg, 2 or more negative test results required to define an infection as having cleared; $\mathrm{Cl}$, confidence intervals; $\mathrm{HIV}$, human immunodeficiency virus; HPV, human papilloma virus; HR, High risk; LR, low risk; MSM, men who have sex with men; MSW, men who have sex with women; NTS, non-type specific; TS, type specific

\section{Acknowledgements}

The authors thank Attila Mihalyi (Global Medical Affairs, Vaccines Value and Health Science at GSK Vaccines) for critically reviewing the manuscript, and Joanne Wolter (independent medical writer, Brisbane, Australia on behalf of GSK Vaccines) who provided writing services and contributed to data extraction and checking. Editing and publication co-ordinating services were provided by Veronique Delpire and Mandy Payne (Words and Science, Brussels, Belgium on behalf of GSK Vaccines). All costs related to the development of this manuscript were met by GlaxoSmithKline Biologicals SA.

\section{Funding}

GlaxoSmithKline Biologicals SA was the funding source and was involved in design and conduct of the study; management, analysis, and interpretation of the data; preparation, review and approval of the manuscript and decision to submit the manuscript for publication. Pallas was involved in the design of the study, conducted the literature review, and review and approval of manuscript. GlaxoSmithKline Biologicals SA funded all costs associated with the development and the publishing of the present manuscript. The corresponding author had full access to the data and was responsible for submission of the publication.

\section{Availability of data and materials}

All data used in this study originates from published studies and are referenced accordingly in this manuscript.

\section{Authors' contribution}

ST conceived the review and ST, EB, MB and XC participated in the design. EB and MB collected and assembled the data, and performed the analysis. ST and XC supervised the analysis. All authors interpreted the data, and reviewed the text. All authors read and approved the final manuscript.

\section{Author's information}

Not applicable.

\section{Competing interests}

ST is an employee of, and owns share options in, the GSK group of companies. $\mathrm{EB}$ and $\mathrm{MB}$ are employees of Pallas health research and consultancy BV, a commercial entity that has received grants and carried out part of the submitted work as a supplier to GSK Vaccines. Pallas has been a supplier of literature reviews and other epidemiological services to GSK Vaccines in the past 36 months. These works cover a variety of vaccines that are in development or on the market.

$X C$ reports having received institutional research and/or educational grants from Merck, Sanofi Pasteur MSD, GSK Vaccines, and Genticel, and occasional personal travel grants and/or speaker fees from Vianex, Merck, and Sanofi Pasteur MSD.

\section{Consent for publication}

Not applicable.

Ethics approval and consent to participate Not applicable.

\section{Author details}

${ }^{1}$ GSK Vaccines, 20, Avenue Fleming, Parc de la Noire Epine, B-1300 Wavre, Belgium. ${ }^{2}$ Pallas Health Research and Consultancy BV, Rotterdam, The Netherlands. ${ }^{3}$ Cancer Epidemiology Research Program, Catalan Institute of Oncology (ICO), IDIBELL, L'Hospitalet de Llobregat, Catalonia, Spain. ${ }^{4} \mathrm{CIBER}$ Epidemiología y Salud Pública (CIBERESP), Barcelona, Spain.

Received: 18 September 2015 Accepted: 7 June 2016

Published online: 14 June 2016

\section{References}

1. Ferlay J, Soerjomataram I, Ervik M, Dikshit R, Eser S, Mathers C, et al. GLOBOCAN 2012 v1.0, Cancer Incidence and Mortality Worldwide: IARC CancerBase No. 11 [Internet]. Lyon, France: International Agency for Research on Cancer. Available from http://globocan.iarc.fr.

2. Cogliano V, Baan R, Straif K, Grosse Y, Secretan B, El Ghissassi F, et al. Carcinogenicity of human papillomaviruses. Lancet Oncol. 2005;6:204.

3. De Vuyst H, Clifford GM, Nascimento MC, Madeleine MM, Franceschi S. Prevalence and type distribution of human papillomavirus in carcinoma and intraepithelial neoplasia of the vulva, vagina and anus: a meta-analysis. Int J Cancer. 2009;124:1626-36. doi:10.1002/ijc.24116.

4. HPV Information Centre. Data Query. 2014. http://www.hpvcentre.net/ dataquery.php (accessed 6 Mar 2015).

5. Mehanna H, Jones TM, Gregoire V, Ang KK. Oropharyngeal carcinoma related to human papillomavirus. BMJ. 2010;340:C1439.

6. de Martel C, Ferlay J, Franceschi S, Vignat J, Bray F, Forman D, et al. Global burden of cancers attributable to infections in 2008: a review and synthetic analysis. Lancet Oncol. 2012;13:607-15. doi:10.1016/S1470-2045(12)70137-7.

7. Herrero R, Quint W, Hildesheim A, Gonzalez P, Struijk L, Katki HA, et al. Reduced prevalence of oral human papillomavirus (HPV) 4 years after bivalent HPV vaccination in a randomized clinical trial in Costa Rica. PLoS ONE. 2013;8:e68329. doi:10.1371/journal.pone.0068329.

8. Giuliano AR, Palefsky JM, Goldstone S, Moreira ED, Penny ME, Aranda C, et al. Efficacy of quadrivalent HPV vaccine against HPV Infection and disease in males. N Engl J Med. 2011;364:401-11. doi:10.1056/NEJMoa0909537.

9. Palefsky JM, Giuliano AR, Goldstone S, Moreira ED, Aranda C, Jessen H, et al. HPV vaccine against anal HPV infection and anal intraepithelial neoplasia. N Engl J Med. 2011;365:1576-85. doi:10.1056/NEJMoa1010971.

10. Lang Kuhs KA, Gonzalez P, Rodriguez AC, van Doorn L-J, Schiffman M, Struijk L, et al. Reduced Prevalence of Vulvar HPV16/18 Infection Among Women Who Received the HPV16/18 Bivalent Vaccine: A Nested Analysis Within the Costa Rica Vaccine Trial. J Infect Dis. 2014;210:1890-9. doi:10.1093/infdis/jiu357.

11. Muñoz N, Kjaer SK, Sigurdsson K, Iversen O-E, Hernandez-Avila M, Wheeler CM, et al. Impact of human papillomavirus (HPV)-6/11/16/18 vaccine on all HPV-associated genital diseases in young women. J Natl Cancer Inst. 2010;102:325-39. doi:10.1093/jnci/djp534. 
12. Kreimer AR, González P, Katki HA, Porras C, Schiffman M, Rodriguez AC, et al. Efficacy of a bivalent HPV 16/18 vaccine against anal HPV 16/18 infection among young women: a nested analysis within the Costa Rica Vaccine Trial. Lancet Oncol. 2011;12:862-70. doi:10.1016/S1470-2045(11)70213-3.

13. McCormack PL. Quadrivalent Human Papillomavirus (Types 6, 11, 16, 18) Recombinant Vaccine (Gardasil( $\left({ }^{\oplus}\right)$ ): A Review of Its Use in the Prevention of Premalignant Anogenital Lesions, Cervical and Anal Cancers, and Genital Warts. Drugs. 2014;74:1253-83. doi:10.1007/s40265-014-0255-z.

14. Schiller JT, Castellsagué $X$, Garland SM. A review of clinical trials of human papillomavirus prophylactic vaccines. Vaccine. 2012;30 Suppl 5:F123-38. doi:10.1016/j.vaccine.2012.04.108

15. D'Souza G, Fakhry C, Sugar EA, Seaberg EC, Weber K, Minkoff HL, et al. Six-month natural history of oral versus cervical human papillomavirus infection. Int J Cancer. 2007;121:143-50. doi:10.1002/ijc.22667.

16. Serwadda D, Wawer MJ, Makumbi F, Kong X, Kigozi G, Gravitt $P$, et al. Circumcision of HIV-infected men: effects on high-risk human papillomavirus infections in a randomized trial in Rakai, Uganda. J Infect Dis. 2010;201:1463-9. doi:10.1086/652185.

17. Backes DM, Snijders PJF, Hudgens MG, Bailey RC, Bogaarts M, Agot K, et al. Sexual behaviour and less frequent bathing are associated with higher human papillomavirus incidence in a cohort study of uncircumcised Kenyan men. Sex Transm Infect. 2013;89:148-55. doi:10.1136/sextrans-2012-050532.

18. Gray RH, Serwadda D, Kong X, Makumbi F, Kigozi G, Gravitt PE, et al. Male circumcision decreases acquisition and increases clearance of high-risk human papillomavirus in HIV-negative men: a randomized trial in Rakai, Uganda. J Infect Dis. 2010;201:1455-62. doi:10.1086/652184

19. Tobian AAR, Kigozi G, Gravitt PE, Xiao C, Serwadda D, Eaton KP, et al. Human papillomavirus incidence and clearance among HIV-positive and HIV-negative men in sub-Saharan Africa. AIDS. 2012;26:1555-65. doi:10.1097/ QAD.0b013e328353b83c

20. Darwich L, Cañadas M-P, Videla S, Coll J, Molina-López RA, Sirera G, et al. Prevalence, clearance, and incidence of human papillomavirus type-specific infection at the anal and penile site of HIV-infected men. Sex Transm Dis. 2013:40:611-8. doi:10.1097/01.OLQ.0000430798.61475.08.

21. Wikström A, Popescu C, Forslund O. Asymptomatic penile HPV infection: a prospective study. Int J STD AIDS. 2000;11:80-4.

22. Mbulawa ZZA, Marais DJ, Johnson LF, Coetzee D, Williamson A-L. Impact of human immunodeficiency virus on the natural history of human papillomavirus genital infection in South African men and women. J Infect Dis. 2012;206:15-27. doi:10.1093/infdis/jis299.

23. Videla S, Darwich L, Cañadas M-P, Coll J, Piñol M, García-Cuyás F, et al. Natural history of human papillomavirus infections involving anal, penile and oral sites among HIV-positive men. Sex Transm Dis. 2013;40:3-10. doi: 10.1097/OLQ.0b013e31827e87bd.

24. Grabowski MK, Gray RH, Serwadda D, Kigozi G, Gravitt PE, Nalugoda F, et al. High-risk human papillomavirus viral load and persistence among heterosexual HIV-negative and HIV-positive men. Sex Transm Infect. 2014;90:337-43. doi:10.1136/sextrans-2013-051230.

25. Giuliano AR, Lu B, Nielson CM, Flores R, Papenfuss MR, Lee J-H, et al. Age-specific prevalence, incidence, and duration of human papillomavirus infections in a cohort of 290 US men. J Infect Dis. 2008;198:827-35. doi:10.1086/591095.

26. Morales R, Parada R, Giuliano AR, Cruz A, Castellsaqué X, Salmerón J, et al. HPV in female partners increases risk of incident HPV infection acquisition in heterosexual men in rural central Mexico. Cancer Epidemiol Biomarkers Prev. 2012;21:1956-65. doi:10.1158/1055-9965.EPI-12-0470.

27. Lajous M, Mueller N, Cruz-Valdéz A, Aguilar LV, Franceschi S, HernándezAvila $\mathrm{M}$, et al. Determinants of prevalence, acquisition, and persistence of human papillomavirus in healthy Mexican military men. Cancer Epidemiol Biomarkers Prev. 2005;14:1710-6. doi:10.1158/1055-9965.EPI-04-0926.

28. Albero $G$, Castellsagué $X$, Lin $H-Y$, Fulp W, Villa LL, Lazcano-Ponce $E$, et al. Male circumcision and the incidence and clearance of genital human papillomavirus (HPV) infection in men: the HPV Infection in men (HIM) cohort study. BMC Infect Dis. 2014;14:75. doi:10.1186/1471-2334-14-75.

29. Partridge JM, Hughes JP, Feng Q, Winer RL, Weaver BA, Xi L-F, et al. Genital human papillomavirus infection in men: incidence and risk factors in a cohort of university students. J Infect Dis. 2007;196:1128-36. doi:10.1086/521192.

30. Kero KM, Rautava J, Syrjänen K, Kortekangas-Savolainen O, Grenman S, Syrjänen S. Stable marital relationship protects men from oral and genital HPV infections. Eur J Clin Microbiol Infect Dis. 2014;33:1211-21. doi:10.1007/ s10096-014-2061-7.
31. Lu B, Wu Y, Nielson CM, Flores R, Abrahamsen M, Papenfuss M, et al. Factors associated with acquisition and clearance of human papillomavirus infection in a cohort of US men: a prospective study. J Infect Dis. 2009;199:362-71. doi:10.1086/596050.

32. Giuliano AR, Harris R, Sedjo RL, Baldwin S, Roe D, Papenfuss MR, et al. Incidence, prevalence, and clearance of type-specific human papillomavirus infections: The Young Women's Health Study. J Infect Dis. 2002;186:462-9. doi:10.1086/341782

33. Glick SN, Feng Q, Popov V, Koutsky LA, Golden MR. High rates of incident and prevalent anal human papillomavirus infection among young men who have sex with men. J Infect Dis. 2014;209:369-76. doi:10.1093/infdis/jit441.

34. Hernandez AL, Efird JT, Holly EA, Berry JM, Jay N, Palefsky JM. Incidence of and risk factors for type-specific anal human papillomavirus infection among HIV-positive MSM. AIDS. 2014;28:1341-9. doi:10.1097/QAD. 0000000000000254

35. Nyitray AG, da Silva RJ C, Baggio ML, D'elle S, Abrahamsen M, Papenfuss M, et al. Six-month incidence, persistence, and factors associated with persistence of anal human papillomavirus in men: the HPV in men study. J Infect Dis. 2011;204:1711-22. doi:10.1093/infdis/jir637.

36. Goodman MT, Shvetsov YB, McDuffie K, Wilkens LR, Zhu X, Ning L, et al. Acquisition of anal human papillomavirus (HPV) infection in women: the Hawaii HPV Cohort study. J Infect Dis. 2008;197:957-66. doi:10.1086/529207.

37. Mullins TLK, Wilson CM, Rudy BJ, Sucharew H, Kahn JA. Incident anal human papillomavirus and human papillomavirus-related sequelae in HIV-infected versus HIV-uninfected adolescents in the United States. Sex Transm Dis. 2013:40:715-20. doi:10.1097/01.olq.0000431049.74390.b7.

38. de Pokomandy A, Rouleau D, Ghattas G, Vézina S, Coté P, Macleod J, et al. Prevalence, clearance, and incidence of anal human papillomavirus infection in HIV-infected men: the HIPVIRG cohort study. J Infect Dis. 2009;199:965-73. doi:10.1086/597207.

39. Shvetsov YB, Hernandez BY, McDuffie K, Wilkens LR, Zhu X, Ning L, et al. Duration and clearance of anal human papillomavirus (HPV) infection among women: the Hawaii HPV cohort study. Clin Infect Dis. 2009:48:536-46. doi:10.1086/596758.

40. Moscicki A-B, Ma Y, Farhat S, Jay J, Hanson E, Benningfield S, et al. Natural history of anal human papillomavirus infection in heterosexual women and risks associated with persistence. Clin Infect Dis. 2014;58:804-11. doi:10.1093/cid/cit947.

41. Goodman MT, Shvetsov YB, McDuffie K, Wilkens LR, Zhu X, Thompson PJ, et al. Prevalence, acquisition, and clearance of cervical human papillomavirus infection among women with normal cytology: Hawaii Human Papillomavirus Cohort Study. Cancer Res. 2008;68:8813-24. doi:10.1158/0008-5472.CAN-08-1380.

42. Moscicki AB, Shiboski S, Broering J, Powell K, Clayton L, Jay N, et al. The natural history of human papillomavirus infection as measured by repeated DNA testing in adolescent and young women. J Pediatr. 1998;132:277-84.

43. Winer RL, Lee S-K, Hughes JP, Adam DE, Kiviat NB, Koutsky LA. Genital human papillomavirus infection: incidence and risk factors in a cohort of female university students. Am J Epidemiol. 2003;157:218-26.

44. Kreimer AR, Pierce Campbell CM, Lin H-Y, Fulp W, Papenfuss MR, Abrahamsen $\mathrm{M}$, et al. Incidence and clearance of oral human papillomavirus infection in men: the HIM cohort study. Lancet. 2013;382:877-87. doi:10.1016/S0140-6736(13)60809-0.

45. Edelstein ZR, Schwartz SM, Hawes S, Hughes JP, Feng Q, Stern ME, et al. Rates and determinants of oral human papillomavirus infection in young men. Sex Transm Dis. 2012;39:860-7. doi:10.1097/OLQ.0b013e318269d098.

46. Kero K, Rautava J, Syrjänen K, Willberg J, Grenman S, Syrjänen S. Smoking increases oral HPV persistence among men: 7-year follow-up study. Eur J Clin Microbiol Infect Dis. 2014;33:123-33. doi:10.1007/s10096-013-1938-1.

47. Darwich L, Cañadas MP, Videla S, Coll J, Molina-López RA, Cobarsi P, et al. Oral human papillomavirus type-specific infection in HIV-infected men: a prospective cohort study among men who have sex with men and heterosexual men. Clin Microbiol Infect. 2014;20:0585-9. doi:10.1111/1469-0691.12523.

48. Kero K, Rautava J, Syrjänen K, Grenman S, Syrjänen S. Oral mucosa as a reservoir of human papillomavirus: point prevalence, genotype distribution, and incident infections among males in a 7-year prospective study. Eur Urol. 2012;62:1063-70. doi:10.1016/j.eururo.2012.06.045.

49. Pickard RKL, Xiao W, Broutian TR, He X, Gillison ML. The prevalence and incidence of oral human papillomavirus infection among young men and women, aged 18-30 years. Sex Transm Dis. 2012;39:559-66. doi:10.1097/ OLQ.0b013e31824f1c65. 
50. Rintala M, Grénman S, Puranen M, Syrjänen S. Natural history of oral papillomavirus infections in spouses: a prospective Finnish HPV Family Study. J Clin Virol. 2006;35:89-94. doi:10.1016/j.jcv.2005.05.012.

51. Louvanto K, Rautava J, Syrjänen K, Grénman S, Syriänen S. The clearance of oral high-risk human papillomavirus infection is impaired by long-term persistence of cervical human papillomavirus infection. Clin Microbiol Infect. 2014;20:1167-72. doi:10.1111/1469-0691.12700.

52. Kurose K, Terai M, Soedarsono N, Rabello D, Nakajima Y, Burk RD, et al. Low prevalence of HPV infection and its natural history in normal oral mucosa among volunteers on Miyako Island, Japan. Oral Surg Oral Med Oral Pathol Oral Radiol Endod. 2004:98:91-6. doi:10.1016/S1079210404000265.

53. Giuliano AR, Lee J-H, Fulp W, Villa LL, Lazcano E, Papenfuss MR, et al. Incidence and clearance of genital human papillomavirus infection in men (HIM): a cohort study. Lancet. 2011;377:932-40. doi:10.1016/501406736(10)62342-2

54. Chelimo C, Wouldes TA, Cameron LD, Elwood JM. Risk factors for and prevention of human papillomaviruses (HPV), genital warts and cervical cancer. J Infect. 2013;66:207-17. doi:10.1016/j.jinf.2012.10.024.

55. Scholefield JH, Castle MT, Watson NFS. Malignant transformation of high-grade anal intraepithelial neoplasia. Br J Surg. 2005;92:1133-6. doi:10.1002/bjs.4994.

56. Stier EA, Sebring MC, Mendez AE, Ba FS, Trimble DD, Chiao EY. Prevalence of anal human papillomavirus infection and anal HPV-related disorders in women: a systematic review. Am J Obstet Gynecol. 2015;213:278-309. doi:10.1016/j.ajog.2015.03.034.

57. Blitzer GC, Smith MA, Harris SL, Kimple RJ. Review of the clinical and biologic aspects of human papillomavirus-positive squamous cell carcinomas of the head and neck. Int J Radiat Oncol Biol Phys. 2014;88:761-70. doi:10.1016/j.jprobp.2013.08.029.

58. Harper DM, Vierthaler SL. Who should be targeted for vaccination against anal cancer? Lancet Oncol. 2011;12:828-9. doi:10.1016/S1470-2045(11)70237-6.

59. Hernandez BY, Shvetsov YB, Goodman MT, Wilkens LR, Thompson P, Zhu X, et al. Reduced clearance of penile human papillomavirus infection in uncircumcised men. J Infect Dis. 2010;201:1340-3. doi:10.1086/651607.

60. Audisio RA, Icardi G, Isidori AM, Liverani CA, Lombardi A, Mariani L, et al. Public health value of universal HPV vaccination. Crit Rev Oncol Hematol. 2016;97:157-67. doi:10.1016/j.critrevonc.2015.07.015.

61. Markowitz LE, Dunne EF, Saraiya M, Chesson HW, Curtis CR, Gee J, et al. Human papillomavirus vaccination: recommendations of the Advisory Committee on Immunization Practices (ACIP). MMWR Recomm Rep. 2014;63:1-30.

62. Australian Government Department of Health and Ageing, National Health and Medical Research Council. The Australian Immunisation Handbook. 10th ed. 2015.

63. Kjaer SK, Munk C, Winther JF, Jørgensen HO, Meijer CJLM, van den Brule AJC Acquisition and persistence of human papillomavirus infection in younger men: a prospective follow-up study among Danish soldiers. Cancer Epidemiol Biomarkers Prev. 2005;14:1528-33. doi:10.1158/1055-9965.EPI-04-0754.

64. Videla S, Darwich L, Cañadas MP, Paredes R, Tarrats A, Castella E, et al. Epidemiological data of different human papillomavirus genotypes in cervical specimens of HIV-1-infected women without history of cervical pathology. J Acquir Immune Defic Syndr. 2009;50:168-75. doi:10.1097/QAI. 0b013e3181938e63.

\section{Submit your next manuscript to BioMed Central and we will help you at every step:}

- We accept pre-submission inquiries

- Our selector tool helps you to find the most relevant journal

- We provide round the clock customer support

- Convenient online submission

- Thorough peer review

- Inclusion in PubMed and all major indexing services

- Maximum visibility for your research

Submit your manuscript at www.biomedcentral.com/submit

) Biomed Central 